\title{
Analytic wavefront sets and operators with multiple characteristics
}

\author{
By Johannes Sjöstrand
}

(Received December 27, 1982)

\section{Introduction}

In this paper we study the analytic wavefront set of solutions of certain differential equations with multiple characteristics. In many situations one can obtain very general results in the analytic theory while the corresponding results in the $C^{\infty}$ theory are much more complicated. As an example we can mention the very general theorem about propagation of analytic singularities for microhyperbolic operators, due to Kashiwara-Kawai [8]. This result only requires a hyperbolicity assumption on the principal symbol of the operator. (The results in the $C^{\infty}$ theory are more complicated, less complete, and depend in general on the lower order symbols of the operator). In [14], [15] we developped some methods to handle situations where only the principal symbol has to be considered. A common point in the various proofs is the inversion of a suitable elliptic problem. Here we will study problems where a reduction to an elliptic problem seems impossible (at least sometimes), and instead we study perturbations of the given problem which are non-elliptic but for which suitable a priori estimates can be obtained.

To be more specific, the class of operators that we shall study (in the analytic case) is the one introduced by Boutet de Monvel, Grigis, Helffer [2]. These authors obtained a very general and satisfactory result concerning the $C^{\infty}$-hypoellipticity with minimal loss of derivatives. On the other hand Trèves [19], Tartakoff [17] and more generally G. Metivier [11] proved the analytic regularity when the characteristic variety is symplectic. We shall give a new proof of Metiviers result. The readers conclusion will hopefully be that the analytic regularity in this case is an easy consequence of facts which are essentially known from the $C^{\infty}$-theory. We will also give some new results in non-symplectic situations. An interesting feature here is the use of Lagrangian manifolds which are only of Lipschitz class. (If we had restricted the attention to Metivier's theorem a shorter proof could certainly have been given). We will also give an extension of a theorem of Ôaku [13]. We beleive that the general method of this paper can and will be 
applied to many other $C^{\infty}$-hypoelliptic operators: (At least in certain cases it should be possible to study the Kohn Laplacian $\square_{b}$, when this operator is h. e. with loss of more than 1 derivative).

In section 1-3 we developp some general arguments. In section 4 we give the various applications and perform the additional geometric arguments required in each particular case. The results of this paper are announced in [16], the last section of [16] gives some remarks that will not be reproduced here.

\section{A priori estimates for a localized operator}

Let $\varphi$ be a real quadratic form on $\boldsymbol{C}^{n}$, strictly plurisub-harmonic (s. pl. s.h.). We also assume for simplicity that $\varphi$ is convex although this last assumption certainly can be eliminated. Let $\Sigma^{C} \subset \boldsymbol{C}^{2 n}=\boldsymbol{C}_{x}^{n} \times \boldsymbol{C}_{\xi}^{n}$ be a complex subspace of codimension $d$, such that $\Sigma=\Sigma^{C} \cap \Lambda_{\varphi}$ is of real codimension $d$ in $\Lambda_{\varphi}$. Here $\Lambda_{\varphi} \subset \boldsymbol{C}^{2 n}$ is the $I$-Lagrangian and $\boldsymbol{R}$-symplectic space given by $\xi=\frac{2}{i} \frac{\partial \varphi}{\partial x}$. (We shall use as much as possible the terminology of [14]). Our last assumption implies that $\Sigma$ is a totally real subspace of maximal dimension in $\Sigma^{c}$.

Let $P=\sum_{|\alpha+\beta| \leqslant m} a_{\alpha \beta} x^{\alpha} D^{\beta}$ be a differential operator of order $m$ in $C^{n}$, $D=\left(\frac{1}{i} \frac{\partial}{\partial x_{1}}, \cdots, \frac{1}{i} \frac{\partial}{\partial x_{n}}\right), a_{\alpha \beta} \in C$. We assume that $P(x, \xi)=\sum_{|\alpha+\beta| \leqslant m} a_{\alpha \beta} x^{\alpha} \xi^{\beta}$ is invariant under all translations parallel to $\Sigma^{c}$, or in other words that $P$ is a symbol on $C^{2 n} / \Sigma^{c}$. The principal symbol in the global sense $p(x, \xi)=$ $\sum_{|\alpha+\beta|=m} a_{\alpha \beta} x^{\alpha} \xi^{\beta}$ is then also invariant under $\Sigma^{c}$-translations and we make the assumption of transversal ellipticity :

$$
p(x, \xi) \neq 0 \quad \text { for } \quad(x, \xi) \in \Lambda_{\varphi} \backslash \Sigma
$$

Let

$$
T u(x)=\int e^{i \varphi(x, y)} u(y) d y
$$

be a "metaplectic" Fourier-Bros-Iagolnitzer transform whose associated linear canonical transformation maps $\boldsymbol{R}^{2 n}$ onto $\Lambda_{\varphi}$. More precisely we require that $\Phi(x, y)$ is a quadratic form on $C_{x}^{n} \times C_{y}^{n}$ such that $\operatorname{det} \Phi_{x y}^{\prime \prime} \neq 0, \operatorname{Im} \Phi_{y y}^{\prime \prime}>0$ and the associated canonical transformation is defined as the map $\mathscr{H}_{T}:\left(y,-\frac{\partial \Phi}{\partial y}\right)$ $\rightarrow\left(x, \frac{\partial \Phi}{\partial x}\right)$. For such a form $\Phi$ we have $\Lambda_{\varphi}=\mathscr{A}_{T}\left(\boldsymbol{R}^{2 n}\right)$ if $\varphi(x)=-\operatorname{Im} \Phi(x, y(x))$. Here $y(x) \in \boldsymbol{R}^{n}$ denotes the minimum point of $y \rightarrow \operatorname{Im} \Phi(x, y)$. That for a 
given st. pl.s. h. quadratic form $\varphi$ we can find a form $\Phi(x, y)$ can be seen as follows : Let $\mathscr{A}$ be an $\boldsymbol{R}$-symplectic linear map $\boldsymbol{R}^{2 n} \rightarrow \Lambda_{\varphi}$ and denote also by $\mathscr{A}$ its $C$-symplectic linear extension $C^{2 n} \rightarrow C^{2 n}$. By $(\overline{y, \eta})$ we denote the ordinary complex conjugate of $(y, \eta)$ and by $(\widehat{x, \xi})$ the complex conjugate of $(x, \xi)$ with respect to $\Lambda_{\varphi}$. If $\mathscr{H}_{T}(y, \eta)=(x, \xi)$ then $\mathscr{H}_{T}(\overline{y, \eta})=(\widehat{x, \xi})$. Hence $\frac{1}{i} \sigma((x, \xi), \widehat{(x, \xi)})=\frac{1}{i} \sigma((y, \eta),(\overline{y, \eta}))$. In [14] we saw that the $C$-Lagrangian plane $x=0$ is strictly negative with respect to $\Lambda_{\varphi}$ i. e. $\frac{1}{i} \sigma((0, \xi),(\widehat{0, \xi}))<0$ for all $\xi \in \boldsymbol{C}^{n}, \xi \neq 0$. On the other hand $\frac{1}{i} \sigma((0, \eta),(0, \bar{\eta}))=0$ so if $\mathscr{A}_{T}((0, \eta))=(0, \xi)$ we have necessarily $\eta=0, \xi=0$. It is then clear that $\mathscr{A}_{T}$ is given by a unique complex quadratic form $\Phi(x, y)$. Since $\{x=0\}$ is st. negative with respect to $\Lambda_{\varphi}$ its preimage $\left\{\eta=-\frac{\partial \Phi(0, y)}{\partial y}\right\}$ is strictly negative with respect to $\boldsymbol{R}^{2 n}$, hence $-\operatorname{Im} \Phi_{y y}^{\prime \prime}<0$.

If $P, \varphi, T$ are as above there exists a unique operator $\tilde{P}=\sum_{|\alpha+\beta| \leqslant m} \tilde{a}_{\alpha \beta} y^{\alpha} D_{y}^{\beta}$ such that $P T u=T \tilde{P} u$ for all $u \in \mathscr{S}\left(\boldsymbol{R}^{n}\right)$. Moreover $P^{w} \circ \mathscr{A}_{T}=\tilde{P}^{w}$ if $P^{w}(x, \xi)$ and $\tilde{P}^{w}(y, \eta)$ denote the Weyl symbols of $P$ and $\tilde{P}$ respectively. Since $P^{w}$ is clearly invariant under $\Sigma^{c}$-translations, $\tilde{P}^{w}$ is invariant under $\tilde{\Sigma}^{c}$-translations where $\tilde{\Sigma}=\mathscr{H}_{T}^{-1}\left(\Sigma^{c}\right)$. Moreover $\tilde{p}=\sum_{|\alpha+\beta|=m} \tilde{a}_{\alpha \beta} y^{\alpha} \eta^{\alpha}=p \circ \mathscr{A}_{T}$ is non-vanishing on $\boldsymbol{R}^{2 n} \backslash \tilde{\Sigma}$, where $\tilde{\Sigma}=\tilde{\Sigma}^{c} \cap \boldsymbol{R}^{2 n}$.

We assume through out this section that $\tilde{P}$ satisfies the equivalent conditions of Theorem 3.1 of Boutet de Monvel-Grigis-Helffer [2]. Then, using also the results of Hörmander [5] there exists a symbol $\tilde{Q}$ on $\boldsymbol{R}^{2 n} / \tilde{\boldsymbol{\Sigma}}$ of order $-m$ such that $\widetilde{Q}_{\#} P^{w}=1=\tilde{P}_{\# \#} \tilde{Q}$ where \# denotes Weyl composition of symbols. Using the stability of the conditions of their Theorem 3.1 that B-G-H also established it is easy to see that $\widetilde{Q}$ admits a holomorphic extension to a domain of the form $\left\{(x+z, \xi+\zeta) ;(x, \xi) \in \boldsymbol{R}^{2 n},(z, \zeta) \in \boldsymbol{C}^{2 n},|(z, \zeta)| \leqslant\right.$ const. $>0\}$ and that the symbols $Q(z+\cdot, \zeta+\cdot \cdot)$ form a bounded set $S^{-m}$ $\left(\boldsymbol{R}^{2 n} / \tilde{\Sigma}\right)$ when $(z, \zeta)$ varies in the ball $|(z, \zeta)| \leqslant$ const.. . Since Weyl-composition of symbols commutes naturally with linear canonical transformations, if we put $Q=\tilde{Q}_{0} \mathscr{H}_{T}^{-1}$, then $Q \# P^{w}=1=P^{w} \# Q$ and $Q$ is holomorphic in a set of the form $\left\{(x+z, \xi+\zeta) ;(x, \xi) \in \Lambda_{\varphi},(z, \zeta) \in C^{2 n},|(z, \zeta)| \leqslant\right.$ const. $\}$, moreover the symbols $Q(z+\cdot, \zeta+\cdot \cdot)$ form a bounded set in $S^{-m}\left(\Lambda_{\varphi} / \Sigma\right)$ when $|(z, \zeta)| \leqslant$ const..

Let $H_{\varphi}$ be the space of entire function on $\boldsymbol{C}^{n}$, square-integrable with respect to $e^{-2 \varphi(x)} L(d x)$, where $L$ is the Lebesque measure on $C^{n}$. When 
working with holomorphic functions having (approximately) this growth at infinity we could try to realize $Q$ as a pseudodifferential operator:

$$
\begin{aligned}
Q u(x) & =\frac{1}{(2 \pi)^{n}} \iint Q\left(\frac{x+y}{2}, \theta\right) e^{i(x-y) \theta} u(y) d y d \theta \\
\theta & =\frac{2}{i} \frac{\partial \varphi}{\partial x}\left(\frac{x+y}{2}\right) .
\end{aligned}
$$

This is no doubt possible since we have $e^{-\varphi(x)+\varphi(y)}\left|e^{i(x-y) \theta}\right|=1$ along the integration contour. For the limited purposes of this paper we rather choose an approximate realization with a slightly better contour:

$$
\begin{gathered}
Q_{R, \chi} u(x)=\frac{1}{(2 \pi)^{n}} \iint Q\left(\frac{x+y}{2}, \theta\right) e^{i(x-y) \theta} \chi\left(\frac{x-y}{R}\right) u(y) d y d \theta \\
\Gamma_{x}: \theta=\frac{2}{i} \frac{\partial \varphi}{\partial x}\left(\frac{x+y}{2}\right)+\frac{i}{R}(\overline{x-y}) .
\end{gathered}
$$

Here $\chi \in C_{0}^{\infty}\left(\boldsymbol{C}^{n}\right)$ is equal to 1 near 0 . If the support of $\chi$ is small enough then $(y, \theta)$ remains in the domain of definition of $Q$ when $(y, \theta) \in \Gamma_{x}, \chi\left(\frac{x-y}{R}\right)$ $\neq 0$.

We shall study $Q_{R, x} \circ P u(x)$, when $u$ is holomorphic in a neighborhood of $\operatorname{supp} \chi\left(\frac{x-\cdot}{R}\right)$. In order to compute $Q_{R, x}\left(D_{y_{j}} u\right)(x)$ we notice that

$$
\begin{aligned}
& \frac{1}{i} d_{(y, \theta)}(\left.\left(\frac{x+y}{2}, \theta\right) e^{i(x-y) \theta} \chi\left(\frac{x-y}{R}\right) u(y)(-1)^{j-1} d y_{1} \wedge \cdots \widehat{d y}_{j} \wedge \cdots \wedge d y_{n} d \theta\right) \\
&=D_{y_{j}}\left(Q\left(\frac{x+y}{2}, \theta\right) e^{i(x-y) \theta} \chi\left(\frac{x-y}{R}\right) u(y) d y d \theta\right. \\
& \quad+Q\left(\frac{x+y}{2}, \theta\right) e^{i(x-y) \theta} \chi\left(\frac{x-y}{R}\right) D_{y_{j}} u d y d \theta \\
& \quad+\sum_{\nu \in \text { finite set }} Q\left(\frac{x+y}{2}, \theta\right) e^{i(x-y) \theta} \frac{1}{R} \chi_{\nu, j}\left(\frac{x-y}{R}\right) \omega_{\nu, j},
\end{aligned}
$$

where $\chi_{\nu, j}$ are first order derivatives of $\chi$ and $\omega_{\nu, j}$ are $2 n$-forms in $(y, \theta)$ with constant coefficients (not necessarily of type $(2 n, 0))$. On $\Gamma_{x}$ we have

$$
d y d \theta=\frac{1}{i^{n}} \operatorname{det}\left(\frac{\partial^{2} \varphi}{\partial \bar{x} \partial x}+\frac{1}{R} I\right) d y d \bar{y},
$$

so $\left.d y d \theta\right|_{\Gamma_{x}}$ is non-degenerate and

$$
\left.\omega_{\nu, j}\right|_{\Gamma_{x}}=\left(\left.\left(c_{\nu, j}+\mathcal{O}\left(\frac{1}{R}\right)\right) d y d \theta\right|_{r_{x}}\right.
$$


where $c_{\nu, j}$ and the term $\mathscr{O}\left(\frac{1}{R}\right)$ are independent of $x, y$.

By Stokes's formula we get

$$
\begin{aligned}
& Q_{R, \chi}\left(D_{y_{j}} u\right)(x)=-\int_{\Gamma_{x}} \int D_{y_{j}}\left(Q\left(\frac{x+y}{2}, \theta\right) e^{i(x-y) \theta} \chi\left(\frac{x-y}{R}\right) u(y) d y \frac{d \theta}{(2 \pi)^{n}}\right. \\
& \quad+\sum_{\nu \in \text { finite set }} \frac{1}{R}\left(c_{\nu, j}+\mathbb{O}\left(\frac{1}{R}\right)\right) Q_{R, x_{\nu}, j} u(x) .
\end{aligned}
$$

Simplifying the first term we get

$$
Q_{R, x}\left(D_{y_{j}} u\right)=\left(Q^{u} \xi_{j}\right)_{R, \chi} u+\sum_{\nu \in f i n i t e \text { set }} \frac{1}{R}\left(c_{\nu, j}+\bigcirc\left(\frac{1}{R}\right)\right) Q_{R, x_{\nu}, j} u
$$

where $\left(Q_{\# \xi_{j}}\right)(x, \xi)=\xi_{j} Q(x, \xi)-\frac{1}{2} D_{x_{j}} Q(x, \xi)$ is the Weyl composition of $Q$ and $\xi_{j}$.

We also compute

$$
\begin{aligned}
Q_{R, x}\left(y_{j} u\right)(x) & =\int_{\Gamma_{x}} \int \frac{\left(x_{j}+y_{j}\right)}{2} Q\left(\frac{x+y}{2}, \theta\right) e^{i(x-y) \theta} \chi\left(\frac{x-y}{R}\right) u(y) d y \frac{d \theta}{(2 \pi)^{n}} \\
& -\int_{\Gamma_{x}} \int Q\left(\frac{x+y}{2}, \theta\right) \frac{\left(x_{j}-y_{j}\right)}{2} e^{i(x-y) \theta} \chi\left(\frac{x-y}{R}\right) u(y) d y \frac{d \theta}{(2 \pi)^{n}}
\end{aligned}
$$

${ }^{2}$ Here the second term is equal to

$$
-\frac{1}{2} \int_{\Gamma_{x}} \int Q\left(\frac{x+y}{2}, \theta\right) D_{\theta_{j}}\left(e^{i(x-y) \theta}\right) \chi\left(\frac{x-y}{R}\right) u(y) d y \frac{d \theta}{(2 \pi)^{n}}
$$

and can be treated as above. We get

$$
Q_{R, \chi}\left(y_{j} u\right)=\left(Q^{\#} x_{j}\right)_{R, \chi} u+\sum_{\nu \in f i n i t e \text { set }} \frac{1}{R}\left(\hat{c}_{\nu, j}+\mathcal{O}\left(\frac{1}{R}\right)\right) Q_{R, \hat{x}_{\nu, j}} u
$$

where $\hat{c}_{\nu, j}$ and the terms $\mathscr{O}\left(\frac{1}{R}\right)$ are independent of $x$ and $\hat{\chi}_{\nu, j}$ denote certain 1 : st order derivatives of $\chi$. The Weyl composition $Q \# x_{j}$ is $x_{j} Q(x, \xi)+$ $\frac{1}{2} D_{\xi_{j}} Q(x, \xi)$.

Let $U_{j}=\sum_{1}^{n} a_{j}^{\nu} x_{\nu}+b_{j}^{\nu} D_{x_{\nu}}, 1 \leqslant j \leqslant d$ be operators with linear symbols $u_{j}=$ $\sum_{1}^{n} a_{j}^{\nu} x_{\nu}+b_{j}^{\nu} \xi_{\nu}$ such that $\Sigma^{c}$ is given by $u_{1}(x, \xi)=\cdots=u_{d}(x, \xi)=\theta$. Then we can write

$$
P=\sum_{k \leqslant m} a_{j_{1}, \ldots, j_{k}} U_{j_{1}} \circ \ldots \circ U_{j_{k}}, a_{j_{1}, \cdots, j_{k}} \in \boldsymbol{C} .
$$

On the other hand 


$$
Q_{R, \chi} U_{j}=\left(Q_{\#}^{\#} U_{j}\right)_{R, \chi}+\sum_{\nu \in f i n i t e ~ s e t} \frac{1}{R}\left(\bar{c}_{\nu, j}+\Theta\left(\frac{1}{R}\right)\right) Q_{R, \hat{x}_{\nu, j}}
$$

(even if we replace $Q$ by an arbitrary symbol which is holomorphic in $\Lambda_{\varphi}+\left\{(z, \zeta) \in C^{2 n} ;|(z, \zeta)| \leqslant\right.$ const. $\left.\}\right)$. By iteration we find

$$
Q_{R, \chi} P=1_{R, \chi}+\sum_{\substack{1 \leqslant j \leqslant m \\(j, k) \in \text { finite set }}} \frac{1}{R^{j}}\left(c_{j, k}+\Theta\left(\frac{1}{R}\right)\right)\left(S_{j, k}\right)_{R, x_{j, k}} .
$$

Here $c_{j, k}$ and the terms $O\left(\frac{1}{R}\right)$ are independent of $x, \chi_{j, k}$ is a derivative of $\chi: \chi_{j, k}=D_{x}^{\alpha} D_{x}^{\beta} \chi,(\alpha, \beta) \neq(0,0)$ and $S_{j, k}$ are symbols with the same domain as $Q$, such that $S_{j, k}(z+\cdot, \zeta+\cdots)$ belong to a bounded set in $S^{-1}\left(\Lambda_{\varphi} / \Sigma\right)$ when $(z, \zeta)$ varies in the ball $|(z, \zeta)| \leqslant$ const..

Let $d_{\Sigma}(x)$ be the Euclidean distance from $\left(x, \frac{2}{i} \frac{\partial \varphi}{\partial x}(x)\right)$ to $\Sigma$. If $\Omega \subset C^{n}$ is open and $u$ is a locally square integrable function on $\Omega$, we put

$$
\begin{aligned}
& \|u\|_{\varphi, \Omega}^{2}=\int_{\Omega} e^{-2 \varphi(x)}|u(x)|^{2} L(d x), \\
& \|u\| \|_{\varphi, \Omega}^{2}=\int_{\Omega} e^{-2 \varphi(x)}\left(1+d_{\Sigma}(x)\right)^{2 m}|u(x)|^{2} L(d x) .
\end{aligned}
$$

Proposition 1.1. Let $P, \varphi$ satisfy all the assumptions above. Then there exists a constant $C>0$ such that for every $R \geqslant 1$ and every function $u$, holomorphic in the ball $B(0,2 R)=\left\{x \in C^{n} ;|x|<2 R\right\}$ :

$$
\|u\|_{\varphi, B(0, R)} \leqslant C\left(\|P u\|_{\varphi, B(0,2 R)}+e^{-R / C}\|u\|_{\varphi, B(0,2 R)}\right) .
$$

ProOF. We take $\chi$ with support in $B\left(0, \frac{1}{2}\right)$ and for $v$ holomorphic in $B(0,2 R)$ we shall first estimate $\left\|Q_{R, \chi} v\right\|_{\varphi, B(0, R)}$.

Let

$$
K(x, y)=Q\left(\frac{x+y}{2}, \theta\right) e^{i(x-y) \theta} \chi\left(\frac{x-y}{R}\right) \operatorname{det} \frac{\partial \theta}{\partial \bar{y}}
$$

be the kernel of $Q_{R, x}\left(\right.$ with $\left.\theta=\frac{2}{i} \frac{\partial \varphi}{\partial x}\left(\frac{x+y}{2}\right)+\frac{i}{R}(\overline{x-y})\right)$. If we are interested in an inequality $\left\|Q_{R, \chi} v\right\|_{\varphi, B(0, R)} \leqslant$ const. $\|v\|_{\varphi, B(0,2 R)}$ we should then consider the reduced kernel

$$
\left(1+d_{\Sigma}(x)\right)^{m} e^{-\varphi(x)+\varphi(y)} e^{i(x-y) \theta} Q\left(\frac{x+y}{2}, \theta\right) \chi\left(\frac{x-y}{R}\right) \operatorname{det} \frac{\partial \theta}{\partial y} .
$$

We split the integral (1.2) in two regions: 


$$
\mathrm{I}:|x-y| \leqslant \frac{R}{C}, \quad \text { II }:|x-y|>\frac{R}{C} \text {. }
$$

Here $C>0$ is large enough so that $\chi\left(\frac{x-y}{R}\right)=1$ in the first region. In the second region the absolute value of the reduced kernel can be estimated by const. $(1+R)^{m} e^{-R / C}$, so with a new constant independent of $v$ and $R \geqslant 1$, we get

$$
\left\|Q_{R, \chi}^{\mathrm{II}} v\right\|_{\varphi, B(0, R)} \leqslant C e^{-R / C}\|v\|_{\varphi, B(0,2 R)}
$$

Here $Q_{R, \chi} v=Q_{R, \chi}^{\mathrm{I}} v+Q_{R, \chi}^{\mathrm{II}} v$ in the obvious way. To estimate $Q_{R, \chi}^{\mathrm{I}} v$ we first replace $\Gamma_{x} \cap\{|x-y| \leqslant R / C\}$ by $\tilde{\Gamma}_{x} \cap\{|x-y| \leqslant R / C\}$, where $\tilde{\Gamma}_{x}$ is the singular contour

$$
\theta=\frac{2}{i} \frac{\partial \varphi}{\partial x}\left(\frac{x+y}{2}\right)+\frac{i}{C} \frac{(\overline{x-y})}{|x-y|}
$$

which coincides with $\Gamma_{x}$ over the sphere $|x-y|=R / C$. (See [14] for the details of this deformation argument). We then get a new reduced kernel whose absolute value can be estimated by a constant times

$$
\tilde{K}(x, y)=\left(1+d_{\Sigma}(x)\right)^{m}\left|Q\left(\frac{x+y}{2}, \theta\right)\right| e^{-|x-y| / C}\left(1+|x-y|^{-n}\right),
$$

where $\theta$ is given by (1.9). Then

$$
\left|Q\left(\frac{x+y}{2}, \theta\right)\right| \leqslant C_{1}\left(1+d_{\Sigma}\left(\frac{x+y}{2}\right)\right)^{-m} \leqslant C_{2}(1+|x-y|)^{m}\left(1+d_{\Sigma}(x)\right)^{-m}
$$

so

$$
\tilde{K}(x, y) \leqslant C_{2}(1+|x-y|)^{m} e^{-|x-y| / C}\left(1+|x-y|^{-n}\right)
$$

and we conclude that

$$
\left\|Q_{R, \chi}^{\mathrm{I}} v\right\|_{\varphi, B(0, R)} \leqslant C\|v\|_{\varphi, B(0,2 R)} .
$$

With (1.8) we get

$$
\left\|Q_{R, \chi} v\right\|_{\varphi, B(0, R)} \leqslant C\|v\|_{\varphi, B(0,2 R)}
$$

for $R \geqslant 1, v$ holomorphic in $B(0,2 R)$. The constant $C$ is independent of $R$ and $v$.

We next study for $u$ holomorphic in $\operatorname{supp} \chi\left(\frac{x-\bullet}{R}\right)$ : 


$$
\begin{aligned}
& 1_{R, x} u(x)=u(x)- \\
& \sum \sum \frac{1}{(2 \pi)^{n}} \int_{\Gamma_{x}} \int_{e^{i(x-y) \theta} g\left((y-x) \cdot\left(\theta-\frac{2}{i} \frac{\partial \varphi}{\partial x}(x)\right)\right)\left(\theta-\frac{2}{i} \frac{\partial \varphi}{\partial x}(x)\right)_{j}} \\
& (-1)^{j+n} u(y) \frac{1}{R} \frac{\partial \chi}{\partial \bar{x}}\left(\frac{x-y}{R}\right) d y \wedge d y \wedge d \theta_{1} \wedge \cdots d \theta_{j} \cdots \wedge d \theta_{n},
\end{aligned}
$$

where

$$
g(t)=-\left(\frac{1}{i t}+\frac{(n-1)}{(i t)^{2}}+\frac{(n-1)(n-2)}{(i t)^{3}}+\cdots+\frac{(n-1) !}{(i t)^{n}}\right) .
$$

In fact, this is formula (12.45) of [14] valid under the assumptions (12.38)(12.40) of that same paper, and in the present case (12.38) and (12.40) are evident while (12.39) follows from some easy calculations :

$$
\begin{gathered}
(y-x) \cdot\left(\theta-\frac{2}{i} \frac{\partial \varphi}{\partial x}(x)\right)=(y-x)\left(\frac{2}{i}\left(\frac{\partial \varphi}{\partial x}\left(\frac{x+y}{2}\right)-\frac{\partial \varphi}{\partial x}(x)\right)\right. \\
\left.+\frac{i}{R}(\overline{x-y})\right)=-i(y-x) \frac{\partial \varphi}{\partial x}(y-x)-\frac{i}{R}|x-y|^{2} .
\end{gathered}
$$

Hence

$$
\operatorname{Im}(y-x) \cdot\left(\theta-\frac{2}{i} \frac{\partial \varphi}{\partial x}(x)\right)=-\varphi(y-x)-\frac{1}{R}|x-y|^{2} \leqslant-\frac{1}{R}|x-y|^{2} .
$$

(This is the only place where we use that $\varphi$ is convex, in the non-convex case, the most efficient way would probably be to prove a substitute for (12. 45), better adapted to the Weyl calculus).

The reduced kernel for the continuity in the \|\|$_{\varphi}$-norm of the general term in the double sum of $(1.11)$ can be estimated by const. $e^{-R / C}$, hence for $u$ holomorphic in $B(0,2 R), R \geqslant 1$ :

$$
\left\|1_{R, \chi} u-u\right\|_{\varphi, B(0, R)} \leqslant C e^{-R / C}\|u\|_{\varphi, B(0,2 R)},
$$

where $C>0$ is independent of $u$ and $R$.

Finally we notice that

$$
\left\|\left(S_{j, k}\right)_{R, x_{j, k}} u\right\|_{\varphi, B(0, R)} \leqslant C e^{-R / C}\|u\|_{\varphi, B(0,2 R)} .
$$

With $v=P u$ we get from (1.10), (1.13) and (1.5):

$$
\left\|1_{R, x} u\right\|_{\varphi, B(0, R)} \leqslant C\left(\|P u\|_{\varphi, B(0,2 R)}+e^{-R / C}\|u\|_{\varphi, B(0,2 R)}\right)
$$

and with (1.12) we get (1.6). This completes the proof.

REMARK 1.2. Let $(P, \varphi, T, \Sigma)=\left(P_{a}, \varphi_{\alpha}, T_{\alpha}, \Sigma_{\alpha}\right)$ be $C^{\infty}$ functions of a 
parameter $\alpha \in M$, where $M$ is a manifold. If the assumptions of Proposition 1. 1. are fulfilled for each $\alpha$, the results of $\mathrm{B}-\mathrm{G}-\mathrm{H}$ on parameter dependence give locally uniform control on the symbol $Q_{\alpha}$ and we conclude that for every compact set $K \subset M$ there is a constant $C>0$ such that (1.6) holds with $P=P_{\alpha}$ for all $R \geqslant 1, \alpha \in K, u$ holomorphic in $B(0,2 R)$.

REMARK 1.3. Naturally Proposition 1.1. still holds if we replace $2 R$ by $\frac{3}{2} R$. If $(\rho, P, \Sigma)$ satisfies the assumptions of the Proposition, $R \geqslant 1$, $\tilde{P}=\sum_{|\alpha+\beta| \leqslant m} \tilde{a}_{\alpha \beta} x^{\alpha} D^{\beta}$ and $\Sigma\left|\tilde{a}_{\alpha \beta}-a_{\alpha \beta}\right|$ is sufficiently small as a function of $R$, then by. Cauchy's inequalities we have $\|(P-\tilde{P}) u\|_{\varphi, B\left(0, \frac{3}{2} R\right)} \leqslant e^{-R / C}\|u\|_{\varphi, B(0,2 R)}$. Then $\tilde{P}$ satisfies (1.6) with a new constant $C>0$, independent of $R$ (although the distance from $\tilde{P}$ to $P$ does depend on $R$ ).

\section{A priori estimates for a pseudodifferential operator}

Let $A\left(x, \tilde{D_{x}}, \lambda\right), \tilde{D}=\frac{1}{\lambda} D_{x}$ be a formal classical analytic pseudodifferential operator of order 0 whose symbol

$$
A(x, \xi, \lambda)=\sum_{0}^{\infty} a_{k}(x, \xi) \lambda^{-k}
$$

is defined in some neighborhood $\widetilde{W}$ of $\left(y_{0}, \eta_{0}\right) \in \boldsymbol{R}^{2 n}$. Let $\tilde{\boldsymbol{\Sigma}}^{c} \subset \boldsymbol{C}^{2 n}$ be a complex submanifold of codimension $d$ such that $\left(y_{0}, \eta_{0}\right) \in \tilde{\Sigma}$ and $\tilde{\Sigma}=\tilde{\Sigma}^{c} \cap \boldsymbol{R}^{2 n}$ is a real analytic submanifold of $\boldsymbol{R}^{2 n}$ of real codimension $d$. Let $m>0$ be an integer and assume as in Boutet-Grigis-Helffer [2] :

$$
\begin{aligned}
& a_{k} \text { vanishes to the order } m-2 k \text { on } \tilde{\Sigma}^{c} \text {, when } m-2 k>0 . \\
& \left|a_{0}\right| \sim d_{\tilde{\Sigma}}^{m} \text { on } \boldsymbol{R}^{2 n} \cap \widetilde{W} \text {, where } d_{\tilde{\Sigma}}(x, \xi) \text { denotes the distance from }
\end{aligned}
$$$$
(x, \xi) \text { to } \tilde{\Sigma} \text {. }
$$

If $(z, \zeta) \in \tilde{\Sigma}$ we put

$$
(A)_{(z, \zeta)}(x, \xi, \lambda)=\sum_{\substack{m-2 k \geqslant 0 \\|\alpha|+|\beta|=m-2 k}} \lambda^{-k} a_{k(\beta)}^{(\alpha)}(z, \zeta)(x-z)^{\beta}(\xi-\zeta)^{\alpha} / \alpha ! \beta !
$$

and

$$
[A]_{(z, \xi)}(y, \eta)=\sum_{\substack{m-2 k \geq 0 \\|\alpha|+|\beta|=m-2 k}} a_{k(\beta)}^{(\boldsymbol{\alpha})}(z, \zeta) y^{\beta} \eta^{\alpha} / \alpha ! \beta !
$$

We can view $[A]_{(z, \xi)}$ as a symbol on $T_{(z, \xi)}\left(\boldsymbol{C}^{2 n}\right)$, easily seen to be invariant under translations along $T_{(z, \xi)}(\tilde{\Sigma})$. We assume

(2.5) For all $(z, \zeta) \in \tilde{\Sigma},[A]_{(z, \zeta)}\left(y, D_{y}\right)$ satisfies the equivalent conditions of Theorem 3.1 of [2]. 
We next perform a FBI-transform. (The explicit choice is without importance).

$$
T u(x, \lambda)=\int e^{-\lambda(x-y)^{2} / 2} u(y) d y .
$$

The associated canonical transformation is $\mathscr{A}_{T}:(y, i(x-y)) \rightarrow(x, i(x-y))$ and $\mathscr{H}_{T}\left(\boldsymbol{R}^{2 n}\right)=\Lambda_{\varphi_{0}}$, where $\varphi_{0}(x)=\frac{1}{2}(\operatorname{Im} x)^{2}$ is convex and strictly pl.s. h.. There is a unique formal classical analytic pseudodifferential operator of order $0 ; P\left(x, \tilde{D}_{x}, \lambda\right)$ defined near $\left(x_{0}, \xi_{0}\right)=\mathscr{A}_{T}\left(y_{0}, \eta_{0}\right) \in \Lambda_{\varphi_{0}}$, such that formally

$$
P T=T A \text {. }
$$

See [14]. We write $P(x, \xi, \lambda)=\sum_{0}^{\infty} p_{k}(x, \xi) \lambda^{-k}$ and define $\Sigma^{c}=\mathscr{A}_{T}\left(\tilde{\Sigma}^{c}\right)$. Let $W$ be some sufficiently small complex neighborhood of $\left(x_{0}, \xi_{0}\right)$. Following Boutet-Grigis-Helffer [2] and Helffer [4] it is easy to establish the following facts.

$$
p_{k} \text { vanishes to the order } m-2 k \text { on } \Sigma \text { when } m-2 k>0
$$

For $(z, \zeta) \in \Sigma$ we define $(P)_{(z, \xi)}$ and $[P]_{(z, \xi)}$ as above. Then if $(z, \zeta)=\mathscr{A}_{\boldsymbol{T}}(\tilde{z}, \tilde{\zeta})$ we have (on $\mathscr{S}\left(\boldsymbol{R}^{n}\right)$ )

$$
[P]_{(z, \xi)} \circ S=S \circ[A]_{(z, \xi)}
$$

where $S$ is the "linearized" FBI transform

$$
S u(x)=\int e^{-(x-y)^{2} / 2} u(y) d y,
$$

whose associated canonical transformation can be identified with the differential of $\mathscr{H}_{T}$ at $(\tilde{\boldsymbol{z}}, \tilde{\zeta})$.

To fix the ideas let us assume that $W$ is of the form $\left|x-x_{0}\right|<a$, $\left|\xi-\xi_{0}\right|<b$ where $\left|\frac{2}{i} \frac{\partial \varphi_{0}}{\partial x}(x)-\xi_{0}\right|<\frac{b}{2}$ for $\left|x-x_{0}\right|<a$. Let $F: W \rightarrow C^{2 n}$ be a Lipschitz mapping, with $F \in C^{0,1}\left(\bar{W} ; C^{2 n}\right):\|F\|_{C^{0,1}(\bar{W})}=\sup _{W}\|F(w)\|+$ $\sup _{W \times W \backslash d} \frac{\|F(w)-F(u)\|}{\|w-\omega\|}<+\infty$. We assumed that $F$ is close to the identity map, i. e. that $\|F-I\|_{0^{0,1}(\bar{W})}$ is small. Then $F$ is bijective $W \rightarrow F(W), F(W)$ is open and the inverse of $F$ is also close to the identity in $C^{0,1}(\overline{F(W)})$. It is clear that $F\left(\Lambda_{\varphi_{0}} \cap W\right)$ is of the form $\xi=H(x)$, where $H \in C^{0,1}\left(\Pi_{x} \circ F\left(W \cap \Lambda_{\varphi_{0}}\right)\right)$. If $\Omega \Subset$ $B\left(x_{0}, a\right)$ is open we will have $\Omega \Subset \Pi_{x} \circ F\left(W \cap \Lambda_{\varphi_{0}}\right)$ and $H$ as close as we like to $\frac{2}{i} \frac{\partial \varphi_{0}}{\partial x}$ in $C^{0,1}\left(\bar{\Omega} ; C^{n}\right)$ provided that $F$ is sufficiently close to the identity 
in $C^{0,1}\left(\bar{W} ; C^{2 n}\right)$. We shall always assume in this section:

(2.12) There exists $\varphi \in C^{1,1}\left(\Pi_{x} \circ F\left(W \cap \Lambda_{\varphi_{0}}\right)\right)$ such that $F\left(W \cap \Lambda_{\varphi_{0}}\right)=\Lambda_{\varphi}$. (2.13) $\quad \Lambda_{\varphi} \cap \Sigma^{c}=F(W \cap \Sigma)$.

Here $C^{1,1}$ is the space of functions of class $C^{1}$ whose first order derivatives are in $C^{0,1}$ and we pick the natural norm. Naturally $\varphi$ is unique up to a constant and if we normalize the choice of $\varphi$ by putting $\varphi\left(x_{0}\right)=\varphi_{0}\left(x_{0}\right)$, if $\Omega \Subset B\left(x_{0}, a\right)$, then $\varphi$ will be as close to $\varphi_{0}$ as we like in $C^{1,1}(\bar{\Omega})$, if $F$ is sufficiently close to the identity in $C^{0,1}(W)$.

Also if $F$ is close to the identity we will still have $\left|p_{0}\right| \sim d_{\Sigma}^{m} c_{\cap \Lambda_{\varphi}}$ on $\Lambda_{\varphi}$, at least if we restrict the attention to a compact subset, as well do. When no confusion is possible we write $d(x)$ instead of $d_{\Sigma} c_{\cap \Lambda_{\varphi}}\left(x, \frac{2}{i} \frac{\partial \varphi}{\partial x}(x)\right)$. If $u$ is a locally square integrable function on $\Omega \Subset B\left(x_{0}, a\right)$ we put

$$
\begin{aligned}
\|u\|_{\varphi, \Omega}^{2} & =\int_{\Omega}|u(x)|^{2} e^{-2 \lambda \varphi(x)} L(d x), \\
\|u\|_{\varphi, \Omega}^{2} & =\int_{\Omega}|u(x)|^{2}\left(\lambda^{-1 / 2}+d(x)\right)^{2 m} e^{-2 \lambda \varphi(x)} L(d x) .
\end{aligned}
$$

Let $L_{\varphi}^{2}(\Omega)$ and $L_{\varphi}^{2, m}(\Omega)$ denote the corresponding spaces.

If $u$ is holomorphic in $\Omega, \Omega_{1} \Subset \Omega$ we can define $P u(x, \lambda)$ for $x \in \Omega_{1}$ by

$$
\begin{aligned}
P u(x, \lambda) & =\left(\frac{\lambda}{2 \pi}\right)^{n} \iint e^{i \lambda(x-y) \theta} P(x, \theta, \lambda) u(y) d y d \theta \\
\theta & =\frac{2}{i} \frac{\partial \varphi}{\partial x}(x)+i T(\overline{x-y}),|x-y| \leqslant r .
\end{aligned}
$$

Here $T>0, r>0$ are independent of $u . \quad r$ is small enough so that dist $\left(\Omega_{1}, C \Omega\right)>r$ and $(x, \theta)$ is well inside $W$, while $T$ is large enough so that

$$
e^{-\lambda(\varphi(x)-\varphi(y))}\left|e^{i \lambda(x-y) \theta}\right| \leqslant e^{-C \alpha|x-y|^{2}}
$$

along the contour, where $C>0 . \quad P(x, \theta, \lambda)$ denotes a realisation of the symbol ([14]). It is then easy to prove that

$$
\|P u\|_{\varphi, \Omega_{1}} \leqslant \text { (const.) }\|u\|_{\varphi, \Omega}
$$

for all $u$ as above and $\lambda \geqslant 1$. Indeed the same type of estimates as we shall develop in the proof of Theorem 2. 1 give (2.17).

THEOREM 2.1. Fix an open set $\Omega_{0} \Subset B\left(x_{0}, a\right)$ and assume that $P, F, \varphi_{0}$, $\varphi$ satisfy all the assumptions above ((2.1), (2.2), (2.5), (2.12), (2.13)). If $F$ is sufficiently close to the identity in the $C^{0,1}$-norm we have the following conclusion: Let $\Omega_{2} \Subset \Omega_{1} \Subset \Omega \Subset \Omega_{0}$ be open sets and define a realisation of $P$ 
as in (2.16). Then there is a constant $C>0$ such that

$$
\|u\|_{\varphi, \Omega_{2}} \leqslant C\left(\|P u\|_{\varphi, \Omega_{1}}+\|u\|_{\varphi, \Omega \backslash \Omega_{2}}\right)
$$

for all $\lambda \geqslant 1$ and all holomorphic functions $u$ on $\Omega$.

Proof. For $\lambda$ in a bounded set, (2.18) follows easily from the maximum principle, so we will have to prove $(2.18)$ only for $\lambda$ large. We shall first prove an a priori inequality in the elliptic region. Write $P=\sum_{m-2 k \geqslant 0} \lambda^{-k} p_{k}(x, \xi)$ $+\mathscr{O}\left(\lambda^{-m / 2}\right)$. We shall estimate

$$
\begin{aligned}
K u(x, \lambda)= & \iint\left(P(x, \eta, \lambda)-p\left(x, \frac{2}{i} \frac{\partial \varphi}{\partial x}\right)\right) e^{i \lambda(x-y) \eta} d y d \eta \\
\eta= & \frac{2}{i} \frac{\partial \varphi}{\partial x}(x)+i T(\overline{x-y}) \\
& |x-y| \leqslant r
\end{aligned}
$$

We split :

$$
P(x, \eta, \lambda)-p\left(x, \frac{2}{i} \frac{\partial \varphi}{\partial x}\right)=\left[p(x, \eta)-p\left(x, \frac{2}{i} \frac{\partial \varphi}{\partial x}\right)\right]+[P(x, \eta, \lambda)-p(x, \eta)]
$$

and write $K u=K_{1} u+K_{2} u$ for the corresponding decomposition of $K u$. By a Taylor expansion to the order $m$ we get

$$
\left|p(x, \eta)-p\left(x, \frac{2}{i} \frac{\partial \varphi}{\partial x}(x)\right)\right| \leqslant C\left(d(x)^{m-1}|x-y|+|x-y|^{m}\right)
$$

and the reduced kernel of $K_{1}$ for the continuity $L_{\varphi}^{2, m}(\Omega) \rightarrow L_{\varphi}^{2}\left(\Omega_{1}\right)$ can be estimated by a constant times

$$
\begin{aligned}
& \frac{\lambda^{n}\left(d(x)^{m-1}|x-y|+|x-y|^{m}\right)}{\left(\lambda^{-1 / 2}+d(y)\right)^{m}} e^{-\lambda|x-y|^{2} / C_{0}} \\
& \quad \leqslant \lambda^{n} \frac{\left(d(x)^{m-1}|x-y|+|x-y|^{m}\right)}{\left(\lambda^{-1 / 2}+d(x)\right)^{m}}\left(1+C \lambda^{1 / 2}|x-y|\right)^{m} e^{-\lambda|x-y|^{2} / C_{0}} \\
& \quad \leqslant \lambda^{n}\left(\frac{|x-y|}{d(x)}+\left(\frac{|x-y|}{d(x)}\right)^{m}\right)\left(1+C \lambda^{1 / 2}|x-y|\right)^{m} e^{-\lambda|x-y|^{2} / C_{0}}
\end{aligned}
$$

Now restrict $x$ to $\Omega_{1, \lambda, R}=\left\{x \in \Omega_{1}: d(x) \geqslant \frac{1}{2} R \lambda^{-1 / 2}\right\}$. Here $R \geqslant 1$ will be chosen large and independent of $\lambda$ later on. Then the reduced kernel of $K_{1}$ can be estimated by

$$
C \lambda^{n} \frac{1}{R}\left(\lambda^{1 / 2}|x-y|+\left(\lambda^{1 / 2}|x-y|\right)^{m}\right)\left(1+C \lambda^{1 / 2}|x-y|\right)^{m} e^{-\lambda|x-y|^{2} / C_{0}}
$$

and we conclude that 
(2.19)

$$
\left\|K_{1} u\right\|_{\varphi, \Omega_{1, R, \lambda}} \leqslant \frac{C}{R}\|u\|_{\varphi, \Omega},
$$

where $C>0$ is independent of $u, \lambda \geqslant 1$ and $R \geqslant 1$.

Let $d_{\Sigma} c(x, \xi)$ be the distance from $(x, \xi)$ to $\Sigma^{c}$. Then for $\eta=\frac{2}{i} \frac{\partial \varphi}{\partial x}(x)$ $+i T(\overline{x-y})$, we have

$$
\begin{gathered}
|P(x, \eta, \lambda)-p(x, \eta)| \leqslant C_{1}\left(\lambda^{-1} d_{\Sigma} c(x, \eta)^{m-2}+\lambda^{-m / 2}\right) \leqslant \\
\leqslant C_{2}\left(\lambda^{-1} d(x)^{m-2}+\lambda^{-m / 2}+\lambda^{-1}|x-y|^{m-2}\right) .
\end{gathered}
$$

The reduced kernel of $K_{2}$ can therefore be estimated by a constant times

$$
\begin{aligned}
& \lambda^{n} \frac{\left(\lambda^{-m / 2}+\lambda^{-1} d(x)^{m-2}+\lambda^{-1}|x-y|^{m-2}\right)}{\left(\lambda^{-1 / 2}+d(y)\right)^{m}} e^{-\lambda|x-y|^{2} / C_{0}} \\
& \leqslant \lambda^{n} \frac{\left(\lambda^{-m / 2}+\lambda^{-1} d(x)^{m-2}+\lambda^{-1}|x-y|^{m-2}\right)}{\left(\lambda^{-1 / 2}+d(x)\right)^{m}}\left(1+C \lambda^{1 / 2}|x-y|^{m}\right) e^{-\lambda|x-y|^{2} / C_{0}}
\end{aligned}
$$

For $x \in \Omega_{1, R, \lambda}$ the last expression is bounded by

$$
\frac{C}{R}\left(1+\left(\lambda^{1 / 2}|x-y|\right)^{m-2}\right)\left(1+\left(\lambda^{1 / 2}|x-y|\right)\right)^{m} e^{-\lambda|x-y|^{2} / C_{0}} \lambda^{n}
$$

so (2.19) is also valid for $K_{2}$. Hence

$$
\|K u\|_{\varphi, \Omega_{1, R, 2}} \leqslant \frac{C}{R}\|u\|_{\varphi, \Omega} .
$$

It is easy to see as in [14] and in the preceeding section that

$$
\|L u\|_{\varphi, \Omega_{1, R, \lambda}} \leqslant C e^{-\lambda / C}\|\boldsymbol{u}\|_{\varphi, \Omega}
$$

if $u$ is holomorphic in $\Omega$ and

$$
\begin{aligned}
L u(x)= & u(x)-\left(\frac{\lambda}{2 \pi}\right)^{n} \iint e^{i \lambda(x-y) \eta} u(y) d y d \eta \\
\eta= & \frac{2}{i} \frac{\partial \varphi}{\partial x}(x)+i T(\overline{x-y}) \\
& |x-y| \leqslant r .
\end{aligned}
$$

Hence with (2.20) we get

$$
\left\|P u-p\left(x, \frac{2}{i} \frac{\partial \varphi}{\partial x}\right) u\right\|_{\varphi, \Omega_{1, R, \lambda}} \leqslant C\left(\frac{1}{R}+e^{-\lambda / C}\right)\|u\|_{\varphi, \Omega},
$$

when $u$ is holomorphic in $\Omega, \lambda \geqslant 1, R \geqslant 1$. Here $C>0$ is independent of $\lambda$ and $R$. Since 


$$
C\left\|p\left(x, \frac{2}{i} \frac{\partial \varphi}{\partial x}(u)\right) u\right\|_{\varphi, \Omega_{1, R, \lambda}} \geqslant\|\boldsymbol{u}\|_{\varphi, \Omega_{1, R, \lambda}}
$$

it follows that

$$
\|u\|_{\varphi, \Omega_{1, R}, \lambda} \leqslant C\left(\|P u\|_{\varphi, \Omega_{1, R}, \lambda}+\left(\frac{1}{R}+e^{-\lambda / C}\right)\|u\|_{\varphi, \Omega}\right) .
$$

Let now $(z, \zeta) \in \Lambda_{\varphi} \cap \Sigma^{c}, z \in \Omega_{1}$ and write $P_{z}(x, \xi, \lambda)$ instead of $P_{(z, \zeta)}(x, \xi, \lambda)$ for the localized symbol, defined as in (2.3). Then

$$
\left|\left(P-P_{z}\right)(x, \xi, \lambda)\right| \leqslant C\left(|x-z|^{m+1}+|\xi-\zeta|^{m+1}+\lambda^{-\frac{m}{2}-\frac{1}{2}}\right) .
$$

If we define $P_{z}$ as a pseudodifferential operator with the same contour as $P$, then for $|x-z| \leqslant 2 R \lambda^{-1 / 2}$, the reduced kernel for the continuity $L_{\varphi}^{2, m}(\Omega) \rightarrow$ $L_{\varphi}^{2}\left(B\left(z, 2 R \lambda^{-1 / 2}\right)\right)$, can be estimated by

$$
\begin{gathered}
C \lambda^{n} R^{m+1} \lambda^{-(m+1) / 2}\left(\lambda^{-1 / 2}+d(y)\right)^{-m} e^{-\lambda|x-y|^{2} / C} \\
\leqslant \tilde{C} R^{m+1} \lambda^{-1 / 2} \lambda^{n} e^{-\lambda|x-y|^{2} / C}
\end{gathered}
$$

(We assume tacitly that $\lambda^{1 / 2} \geqslant \frac{1}{\varepsilon_{0}} R$ where $\varepsilon_{0}>0$ is small and fixed).

Let $x_{1}, \cdots, x_{N} \in \Pi_{x}\left(\Sigma^{C} \cap \Lambda_{\varphi}\right) \cap \Omega_{1}$ have the property that no point $x$ is in more than $N_{0}$ of the balls $B\left(x_{j}, 2 R \lambda^{-1 / 2}\right)$. If $\stackrel{N}{D} U B\left(x_{j}, 2 R \lambda^{-1 / 2}\right)$ denotes the disjoint union of these balls and $\varphi$ is considered also as a function on this set, we can identify unitarily:

$$
\bigoplus_{1}^{N} L_{\varphi}^{2}\left(B\left(x_{j}, 2 R \lambda^{-1 / 2}\right)\right) \simeq L_{\varphi}^{2}\left(D_{1}^{N} B\left(x_{j}, 2 R \lambda^{-1 / 2}\right)\right) .
$$

With this identification the map

$$
L_{\varphi}^{2, m}(\Omega) \ni u \rightarrow\left(\left(P-P_{x_{1}}\right) u, \cdots,\left(P-P_{x_{N}}\right) u\right) \in \bigoplus_{1}^{N} L_{\varphi}^{2}\left(B\left(x_{j}, 2 R \lambda^{-1 / 2}\right)\right)
$$

has a reduced kernel $K(x, y)$, that we can estimate by (2.24). Then

$$
\begin{aligned}
& \int_{D U}|K(x, y)| L(d x) \leqslant C N_{0} \lambda^{-1 / 2} R^{m+1} \\
& \int_{\Omega}|K(x, y)| L(d y) \leqslant C \lambda^{-1 / 2} R^{m+1},
\end{aligned}
$$

so the norm of the map (2.25) is at most $C \lambda^{-1 / 2} \sqrt{N_{0}} R^{m+1}$.

Let $\bar{P}_{x_{j}} u=P_{x_{j}}\left(x, \frac{D_{x}}{\lambda}, \lambda\right) u$ be $P_{x_{j}}$ as a differential operator.

Then as in [14] we see that 


$$
\left\|\left(P_{x_{j}}-\bar{P}_{x_{j}}\right) u\right\|_{\varphi, B\left(x_{j}, 2 R \lambda^{-1 / 2}\right)} \leqslant C e^{-\lambda / C}\|u\|_{\varphi, \Omega} .
$$

The result of the preceesing section gives

LEMMA 2.2. There is a constant $C>0$ such that for every $R \geqslant 1$, if $F$ is close enough to the identity as a function of $R$, then for all $\lambda$ large enough and all $z \in \Pi\left(\Lambda_{\varphi} \cap \Sigma^{c}\right) \cap \Omega_{1}$, we have

$$
\|u\|_{\varphi, B\left(z, R \lambda^{-1 / 2}\right)} \leqslant C\left(\left\|\bar{P}_{z} u\right\|_{\varphi, B\left(z, 2 R \lambda^{-1 / 2}\right)}+e^{-R / C}\|u\|_{\varphi, B\left(z, 2 R \lambda^{-1 / 2}\right.}\right)
$$

when ever $u$ is a holomorphic function in $B\left(z, 2 R \lambda^{-1 / 2}\right)$.

Proof. Let $U$ be the operator $u=u(x) \rightarrow v(t)$ where $u=e^{i \lambda(x-z) \xi} v$, $x=z+\lambda^{-1 / 2} t$. Then

$$
U \circ\left(\tilde{D}_{x}-\zeta\right)=\lambda^{-1 / 2} D_{t} \circ U, U \circ((x-z) \cdot)=\left(\lambda^{-1 / 2} t \cdot\right) \circ U,
$$

so $U \circ P_{z}=\lambda^{-m / 2}[P]_{z} \circ U$. If $\Phi(t)=\lambda(\varphi(x)-\langle\nabla \varphi(z),(x-z)\rangle), t=\lambda^{1 / 2}(x-z)$, then

$$
|u(x, \lambda)| e^{-\lambda \varphi(x)}=|v(t, \lambda)| e^{-\Phi(t)}
$$

so

$$
\|u\|_{\varphi, B\left(z, R \lambda^{-1 / 2}\right.}=\lambda^{-n / 2}\|v\|_{\Phi, B(0, R)}
$$

and similarly with $R$ replaced by $2 R$. (The norm to the right is defined as in section 1).

If $\|F-I\|_{c^{0,1}}=\mathscr{O}(\varepsilon)$, then $\nabla \varphi(x+h)-\nabla \varphi(x)=\left(\nabla^{2} \varphi_{0}+\mathscr{O}(\varepsilon)\right) h$, hence $\Phi(t)=$ $\varphi_{0}(t)+\mathscr{O}(\varepsilon) t^{2}$ and so for instance

$$
\|v\|_{\phi, B(0, R)}=\left(1+\mathscr{O}_{R}(\varepsilon)\right)\|\boldsymbol{v}\|_{\varphi_{0}, B(0, R)}
$$

where $\mathscr{O}_{R}(\varepsilon)$ indicates a term which in absolute value is bounded by $C_{R} \cdot \varepsilon$. Here $C_{R}$ is independent of $\lambda, v$. Let $(z, \zeta)=F(\tilde{z}, \tilde{\zeta}),(x, \xi)=F(\tilde{x}, \tilde{\xi}) \in \Lambda_{\varphi}$. If $d_{\varphi_{0}}$ denotes the distance to $\Lambda_{\varphi_{0}} \cap \Sigma^{c}=\Sigma$ and $d_{\varphi}$ that to $\Lambda_{\varphi} \cap \Sigma^{c}$, we have $d_{\varphi}(x, \xi)=(1+\mathscr{O}(\varepsilon)) d_{\varphi_{0}}(\tilde{x}, \tilde{\xi})$. Assume in addition that $|x-z| \leqslant 2 R \lambda^{-1 / 2}$, and let us remember that $x-z=(I+\mathscr{O}(\varepsilon))(\tilde{x}-\tilde{z})$. The point $(y, \eta)$ in $\Sigma$ which realizes the distance $d_{\varphi_{0}}(\tilde{x}, \tilde{\xi})$ is at a distance at most (const.) $R \lambda^{-1 / 2}$ from $(\tilde{z}, \tilde{\zeta})$. Hence $d\left((y, \eta), \quad(\tilde{z}, \widetilde{\zeta})+T_{(z, \tilde{\xi})}(\Sigma)\right)=\mathscr{O}\left(R^{2} \lambda^{-1}\right)$. Similarly, if $\left(y^{\prime}, \eta^{\prime}\right) \in$ $(\tilde{z}, \tilde{\zeta})+T_{(\tilde{z}, \tilde{\xi})}(\Sigma)$ is at minimal distance to $(x, \xi)$ and $\left|\left(y^{\prime}, \eta^{\prime}\right)-(\tilde{z}, \tilde{\zeta})\right|=\mathscr{O}\left(\mathrm{R} \lambda^{-1 / 2}\right)$, then $\left.d_{\varphi_{0}}\left(y^{\prime}, \eta^{\prime}\right)\right)=\mathscr{O}\left(R^{2} \lambda^{-1}\right)$. It follows that

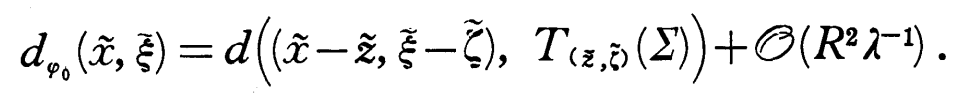

Now write $(\tilde{x}-\tilde{z}, \tilde{\xi}-\tilde{\zeta})=\lambda^{-1 / 2}(\tilde{t}, \tilde{\tau})$, where $\tilde{\tau}=\frac{2}{i} \frac{\partial \varphi_{0}}{\partial \tilde{t}}(\tilde{t})$ and $t=(I+\mathscr{O}(\varepsilon)) \tilde{t}$. Then 


$$
d_{\varphi_{0}}(\tilde{x}, \tilde{\xi})=\lambda^{-1 / 2} d\left((t, \tau), T_{(z, \tilde{z})}(\Sigma)\right)+\mathscr{O}\left(\varepsilon R \lambda^{-1 / 2}+R^{2} \lambda^{-1}\right)
$$

if $\tau=\frac{2}{i} \frac{\partial \varphi_{0}}{\partial t}(t)$. Hence for $\varepsilon>0$ small enough and $\lambda$ large enough as a function of $R$ and $\varepsilon$ we get

$$
\lambda^{-1 / 2}+d_{\varphi}(x, \xi)=\left(1+\mathscr{O}_{R}(\varepsilon)\right) \lambda^{-1 / 2}\left(1+d\left((t, \tau), T_{(z, \tilde{\xi})}(\Sigma)\right)\right)
$$

when $\xi=\frac{2}{i} \frac{\partial \varphi}{\partial x}, t=\lambda^{1 / 2}(x-z), \tau=\frac{2}{i} \frac{\partial \varphi_{0}}{\partial t}(t),|x-z| \leqslant 2 R^{-1 / 2}$. We then get

$$
\|u\|_{\varphi, B\left(z, R \lambda^{-1 / 2}\right)}=\lambda^{-m / 2-n / 2}\left(1+\mathscr{O}_{R}(\varepsilon)\right)\|v\|_{\varphi_{0}, B(0, R)}
$$

where the norm to the right is defined as in section 1 . Since $(z, \zeta)=(\tilde{z}, \tilde{\zeta})+$ $\mathcal{O}(\varepsilon)$ we can consider $[P]_{z}$ as a small perturbation of $[P]_{z}$. Proposition 1.1 and the Remarks 1.2 and 1.3 then give

$$
\|v\|_{\varphi_{0}, B(0, R)} \leqslant C\left(\left\|[P]_{z} v\right\|_{\varphi_{0}, B(0,2 R)}+e^{-R / C}\|v\|_{\varphi_{0}, B(0,2 R)}\right)
$$

when $\varepsilon$ is small enough as a function of $R$. Using the equivalence of the various norms for $v=U u$ and $u$ as well as the fact that $[P]_{z} v=\lambda^{m / 2} U P_{z} u$ we get (2.27) and the Lemma is proved.

When $F$ is sufficiently close to the identity as a function of $R$ and $\lambda$ is large enough, we get:

$$
\begin{aligned}
& \sum_{1}^{N}\|u\|_{\varphi, B\left(x_{j}, R \lambda^{-1} / 2\right)}^{2} \leqslant \\
& \leqslant C \sum_{1}^{N}\left\|\bar{P}_{x_{j}} u\right\|_{\varphi, B\left(x_{j}, 2 R \lambda^{-1 / 2}\right)}^{2}+e^{-R / C}\|u\|_{\varphi, B\left(x_{j}, 2 R \lambda^{-1 / 2}\right)}^{2} \\
& \leqslant 2 C \sum_{1}^{N}\|P u\|_{\varphi, B\left(x_{j}, 2 R \lambda^{-1 / 2}\right)}^{2}+2 C \sum_{1}^{N}\left\|\left(P_{x_{j}}-P\right) u\right\|_{\varphi, B\left(x_{j}, 2 R \lambda^{-1 / 2}\right)}^{2} \\
& \quad+C N e^{-\lambda / C}\|u\|_{\varphi, \Omega}^{2}+C \sum_{1}^{N} e^{-R / C}\|u\|_{\varphi, B\left(x_{j}, 2 R \lambda^{-1 / 2}\right)}^{2}
\end{aligned}
$$

Here we use the estimate of the norm of $(2.25)$ to get

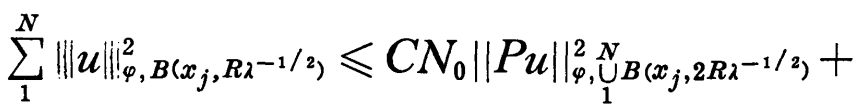

$$
\begin{aligned}
& +\left(C \lambda^{-1} N_{0} R^{2(m+1)}+C N e^{-\lambda / C}+C N_{0} e^{-R / C}\right)\|u\|_{\varphi, \Omega}^{2} .
\end{aligned}
$$

This estimate is valid with a constant $C>0$ independent of $R, x_{1}, \cdots, x_{N}, \lambda$, $u$, provided that $F$ is close enough to the identity as a function of $R$ and $\lambda$ sufficiently large as a function of $R$. We now claim that there exist $C_{0}$, $N_{0}>0$ such that for $\lambda \geqslant 1, R \geqslant 1,4 R \lambda^{-1 / 2}<\min \left(r, d\left(\Omega_{2}, C \Omega_{1}\right)\right)$ we can find $x_{1}, \cdots, x_{N} \in \Pi\left(\Lambda_{\varphi} \cap \Sigma^{C}\right) \cap\left(\Omega_{2}+B\left(0, R \lambda^{-1 / 2}\right)\right)$ such that $N \leqslant C_{0} \lambda^{n}$, 


$$
\bigcup_{1}^{N} B\left(x_{j}, R \lambda^{-1 / 2}\right) \supseteq\left\{x \in \Omega_{2} ; d\left(x, \Pi\left(\Sigma^{c} \cup \Lambda_{\varphi}\right)\right) \leqslant \frac{1}{2} R \lambda^{-1 / 2}\right\}=\Omega_{2} \backslash \Omega_{1, R, \lambda},
$$

such that no point is in more than $N_{0}$ of the balls $B\left(x_{j}, 2 R \lambda^{-1 / 2}\right)$. In fact, we cut the space into closed cubes with disjoint interiors of diagonal $\frac{1}{4} R \lambda^{-1 / 2}$. For each such cube which intersects $\Pi\left(\Sigma^{c} \cap \Lambda_{\varphi}\right) \cap\left(\Omega_{2}+B\left(0, R \lambda^{-1 / 2}\right)\right)$, we take a point $x_{j}$ in the intersection. The above properties of the covering are then almost immediate.

With such a covering (2.29) implies (with a new constant $C$ ):

$$
\|u\|_{\varphi, \Omega_{2} \backslash \Omega_{1, R, \lambda}}^{2} \leqslant C\left(\|P u\|_{\varphi, \Omega_{1}}^{2}+\left(\lambda^{-1} R^{2(m+1)}+e^{-\lambda / C}+e^{-R / C}\right)\|u\|_{\varphi, \Omega}^{2}\right) .
$$

This with (2.22) gives

$$
\|u\|_{\varphi, \Omega_{2}}^{2} \leqslant C\left(\|P u\|_{\varphi, \Omega_{1}}^{2}+\left(\frac{1}{R}+\lambda^{-1} R^{2(m+1)}+e^{-\lambda / C}+e^{-R / C}\right)\|\boldsymbol{u}\|_{\varphi, \Omega}^{2}\right)
$$

Here we chose successively $R$ and $\lambda$ large enough so that

$$
C\left(\frac{1}{R}+\lambda^{-1} R^{2: m+1)}+e^{-\lambda / C}+e^{-R / C}\right) \leqslant \frac{1}{2}
$$

and (2.18) follows. The proof of Theorem 2.1 is complete.

Remark 2.3. If $P^{\prime}$ is another realisation of $P$ such that $\left\|P u-P^{\prime} u\right\|_{\varphi_{,} \Omega_{1}}$ $\leqslant \frac{1}{\varepsilon} e^{-\varepsilon \lambda}\|u\|_{\varphi, \Omega}$ for some $\varepsilon>0$ independent of $\lambda$, then from (2.18) we see that (2.18) is also valid with $P$ replaced by $P^{\prime}$, provided that we change the constant $C$.

\section{Some geometric preparations}

We shall first verify that the standard local Hamilton-Jacobi theory is valid when the Hamiltonian is of class $C^{1,1}$. The Hamilton field is then of class $C^{0,1}$ and we start by recalling some easy estimates for $C^{0,1}$ vector fields. Let $\Omega \subset \boldsymbol{R}^{n}$ be an open set and $v \in C^{0,1}\left(\bar{\Omega} ; \boldsymbol{R}^{n}\right)$ a Lipschitz vector field. If $K \subset \Omega$ is compact there is an $\varepsilon>0$ such that the problem $\dot{x}(t)=v(x(t))$, $x(0)=x_{0}, 0 \leqslant t \leqslant \varepsilon$ has a unique solution for every $x_{0} \in K$. Take now two Lipschitz fields $v_{1}$ and $v_{2}$ on $\bar{\Omega}$ with $\left\|v_{j}\right\|_{\mathcal{C}^{0,1}(\bar{Q})} \leqslant C,\left\|v_{1}-v_{2}\right\|_{\mathcal{C}^{\circ}(\bar{i})} \leqslant D$, and consider two integral curves $\dot{x}_{j}(t)=v_{j}\left(x_{j}((t))\right.$ (always assumed to stay in some fixed compact set that we do not specify). Then

$$
\dot{x}_{1}(t)-\dot{x}_{2}(t)=\left(v_{1}\left(x_{1}(t)\right)-v_{1}\left(x_{2}(t)\right)\right)+\left(v_{1}\left(x_{2}(t)\right)-v_{2}\left(x_{2}(t)\right)\right.
$$

so 


$$
\left|\dot{x}_{1}(t)-\dot{x}_{2}(t)\right| \leqslant C\left|x_{1}(t)-x_{2}(t)\right|+D,
$$

which implies

$$
\left|x_{1}(t)-x_{2}(t)\right| \leqslant e^{C t}\left|x_{1}(0)-x_{2}(0)\right|+D \frac{\left(e^{C t}-1\right)}{C} .
$$

If we let $\Phi_{j, t}: x_{j}(0) \rightarrow x_{j}(t)$ be the flow of $v_{j}$ (defined for $x_{j}(0) \in K, 0 \leqslant t \leqslant \varepsilon_{K}$ $>0)$, (3.2) shows that

$$
\left\|\Phi_{1, t}-\Phi_{2, t}\right\|_{C^{0}} \leqslant D \frac{\left(e^{C^{0}}-1\right)}{C} .
$$

Suppose that $v_{1}=v_{2}=v,\|v\|_{c^{0,1}} \leqslant C$. Then (3.2) gives

$$
\left|x_{1}(t)-x_{2}(t)\right| \leqslant e^{C t}\left|x_{1}(0)-x_{2}(0)\right|
$$

and (3.1) gives

$$
\left|\dot{x}_{1}(t)-\dot{x}_{2}(t)\right| \leqslant C e^{C t}\left|x_{1}(0)-x_{2}(0)\right| .
$$

Hence

$$
\left|\left(x_{1}(t)-x_{2}(t)\right)-\left(x_{1}(0)-x_{2}(0)\right)\right| \leqslant\left(e^{C t}-1\right)\left|x_{1}(0)-x_{2}(0)\right| .
$$

Since $|x(t)-x(0)| \leqslant C t$ we get

$$
\| \Phi_{t}-I||_{0^{0,1}} \leqslant 2\left(e^{C t}-1\right),
$$

where $\Phi_{t}(x(0))=x(t)$ is the flow of $v$. Using the group property we also get $\left\|\Phi_{t}^{-1}-I\right\|_{c^{0,1}} \leqslant 2\left(e^{C t}-1\right)$.

Let $p \in C^{1,1}(\bar{W} ; \boldsymbol{R})$ where $W$ now is an open set in $\boldsymbol{R}^{2 n}$. Then we can apply the above estimates to $H_{p} \in C^{0,1}\left(W ; \boldsymbol{R}^{2 n}\right)$. Let $\Omega \subset \boldsymbol{R}^{n}$ be an open ball $\varphi_{0} \in C^{2}(\bar{\Omega} ; \boldsymbol{R})$ and assume that $\Lambda_{0}=\left\{\left(x, \frac{\partial \varphi_{0}}{\partial x}\right) ; x \in \Omega\right\} \Subset W$. We can then define $\Lambda_{t}=\Phi_{t}\left(\Lambda_{0}\right)$ for $0 \leqslant t \leqslant \varepsilon>0$, if $\Phi_{t}$ is the flow of $H_{p}$. For $t$ small enough, $\Lambda_{t}$ will be of the form $\xi=h_{t}(x)$ where $h_{t} \in C^{0,1}\left(\Pi_{x}\left(\Lambda_{t}\right)\right)$ and if $K \Subset \Omega$ we have $K \subset \Pi_{x}\left(\Lambda_{t}\right)$ when $t$ is small enough. Let $\chi \in C_{0}^{\infty}\left(\boldsymbol{R}^{2 n}\right)$ with $\int \chi d x d \xi=1, \chi_{\mathbf{s}}=$ $\varepsilon^{-2 n} \chi(x / \varepsilon, \xi / \varepsilon)$. After decreasing $W$ slightly we can define $p_{\varepsilon}=\chi_{\varepsilon} * p$ on $W$ for $0 \leqslant \varepsilon \leqslant \varepsilon_{0}$ and $\left\{p_{\varepsilon}\right\}$ is a bounded family in $C^{2}$ while $p_{t} \rightarrow p$ in $C^{1}$. Hence $H_{p} \rightarrow$ $H_{p}$ in $C^{0}(W)$ and (3.3) shows that $\Phi_{t} \rightarrow \Phi_{t}$ in $C^{0}$ for the corresponding flows, uniformly for $t$ in some small interval $\left[0, t_{0}\right]$. On the other hand $\Lambda_{i}^{i}=\Phi_{t}^{i}\left(\Lambda_{0}\right)$ is of the form $\xi=h_{t}^{\varepsilon}(x)$ with $h_{t}^{i} \in C^{0,1}$ forming a bounded family (when restricted to $K(\varepsilon)$ and $h_{t} \rightarrow h_{t}$ in $C^{0}$ uniformly in $t$ when $\varepsilon \rightarrow 0$. Now $\Lambda_{t}^{i}$ is of the form $\xi=\frac{\partial \varphi_{s}(t, x)}{\partial x}$ where $\varphi_{s} \in C^{2}$ solves the characteristic equation 
$\frac{\partial \varphi_{\mathrm{s}}}{\partial t}+p_{\mathrm{s}}\left(x, \frac{\partial \varphi_{\mathrm{s}}}{\partial x}(t, x)\right)=0,0 \leqslant t \leqslant t_{0}, x \in \Pi_{x}\left(\Lambda_{t}^{\mathrm{z}}\right)$, with $\varphi_{\mathrm{c}}(0, x)=\varphi_{0}(x)$. It is then clear that $\varphi_{\mathrm{s}}$ converges in $C^{1}\left(\left[0, \varepsilon_{K}\right] \times K\right)$ as $\varepsilon \rightarrow 0$. If $\varphi$ is the limit we have $\frac{\partial \varphi}{\partial x}(t, x)=h_{t}(x)$ and in the limit we get

$$
\frac{\partial \varphi}{\partial t}+p\left(x, \frac{\partial \varphi}{\partial x}\right)=0,\left.\varphi\right|_{t=0}=\varphi_{0} \text {. }
$$

Clearly $\varphi \in C^{1,1}$. It is not hard to show that $\left\|\varphi(t, \cdot)-\varphi_{0}\right\|_{C^{1,1}(K)} \rightarrow 0$ as $t \rightarrow 0$. (It is essential that $\varphi_{0} \in C^{2}$, the proof is particularly simple when $\varphi_{0}$ is a second order polynomial so that $\Lambda_{0}$ is linear and we shall only use this particular case below).

We now translate the preceeding discussion into the "I-Lagrangian" terminology. Let $r \in C^{1,1}(W ; \boldsymbol{C})$ where $W$ is an open neighborhood of $\left(x_{0}, \xi_{0}\right) \in C^{2 n}$. Let $\varphi_{0} \in C^{2}(\Omega ; \boldsymbol{R})$ where $\Omega$ is an open neighborhood of $x_{0}$ and assume that $\Lambda_{\varphi_{0}}=\left\{\left(x, \frac{2}{i} \frac{\partial \varphi_{0}}{\partial x}(x)\right) ; x \in \Omega\right\} \subset W$. Then after replacing $\Omega$ by a relatively compact open subset we can solve the problem

$$
\frac{\partial \varphi}{\partial t}-(\operatorname{Re} r)\left(x, \frac{2}{i} \frac{\partial \varphi}{\partial x}\right)=0, \varphi(0, x)=\varphi_{0}(x)
$$

for $x \in \Omega, 0 \leqslant t \leqslant \varepsilon_{0}>0$ and the solution is of class $C^{1,1}([0, \varepsilon] \times \Omega ; \boldsymbol{R})$. The real symplecticfo $\mathrm{rm}$ we use here is $d \tau \wedge d t-\operatorname{Im} d \xi \wedge d x$, and $\Lambda_{\varphi_{t}}=\left\{\left(x, \frac{2}{i} \frac{\partial \varphi}{\partial x}(t, x)\right)\right.$; $x \in \Omega\}$ is equal to $\exp \left(t H_{\mathrm{Re} r}^{\operatorname{Im} \sigma}\right)\left(\Lambda_{\varphi_{0}}\right)$. Here $H_{\mathrm{Re} r}^{\operatorname{Im} \sigma}$ denotes the Hamilton field of $\operatorname{Re} r$ with respect to $\operatorname{Im} \sigma=\operatorname{Im} d \xi \wedge d x$. We refer to [14] for more details. At a point where $\bar{\partial} r=0$ we have $H_{\mathbf{R e r}}^{\operatorname{Im} \sigma}=\widehat{H_{i r}}$, where $H_{i r}$ is the complex standard Hamilton field of ir (of type $(1,0)$ ) and $H_{i r}$ is the associated real vector field which gives the same result when applied to holomorphic functions in $(x, \xi)$. If $r$ is holomorphic in $W$ we can actually solve the problem

$$
2 \frac{\partial \varphi}{\partial t}-r\left(x, \frac{2}{i} \frac{\partial \varphi}{\partial x}\right)=0, \varphi(0, x)=\varphi_{0}(x)
$$

for $t \in C,|t| \leqslant \varepsilon_{0}$, where $\frac{\partial}{\partial t}=\frac{1}{2}\left(\frac{\partial}{\partial \operatorname{Re} t}+\frac{1}{i} \frac{\partial}{\partial \operatorname{Im} t}\right)$ and the solution of (3.6) is then the restriction to the positive real axis of the solution of (3.7). $\Lambda_{\varphi_{t}}$, defined as before, is now equal to $\exp \left(\widehat{i t H_{r}}\right)\left(\Lambda_{\varphi_{0}}\right)$.

We assume from now on that $r$ is real valued on $\Lambda_{\varphi_{0}}$ and that $\bar{\partial} r=0$ at every point of $\Lambda_{\varphi_{0}}$. A compact set $H \subset \Lambda_{\varphi_{0}}$ will be called a barrier if there is a constant $C>0$ such that 


$$
|\operatorname{Vr}(x, \xi)|^{2} \leqslant \operatorname{Cr}(x, \xi) \quad \text { for all } \quad(x, \xi) \in H .
$$

If $H$ is such a barrier and $\delta_{0}>0$ is a sufficiently small constant, then for $(x, \xi) \in H,|(y, \eta)-(x, \xi)| \leqslant \delta_{0}|\operatorname{Vr}(x, \xi)|$ we have

$$
|\operatorname{Vr}(y, \eta)|=\left(1+\mathbb{O}\left(\delta_{0}\right)\right)|\operatorname{Vr}(x, \xi)|, \operatorname{Re} r(y, \eta)=\left(1+\mathscr{O}\left(\delta_{0}\right)\right) r(x, \xi)
$$

and in particular $\operatorname{Re} r(y, \eta) \geqslant 0$. If $\varepsilon_{0}>0$ is small enough, $0 \leqslant t \leqslant \varepsilon_{0}$ and $(x, \xi)$ $\in H$, then $\exp \left(-t H_{\mathrm{Re} r}^{\operatorname{Im} q}\right)\left(\Lambda_{\varphi_{0}} \cap B\left((x, \xi), \frac{1}{2} \delta_{0}|\nabla r(x, \xi)|\right)\right) \subset B\left((x, \xi), \delta_{0}|\operatorname{Vr}(x, \xi)|\right)$ and the projection of this set contains $x$. Hence $\operatorname{Re} r\left(x, \frac{\partial \varphi_{t}(x)}{\partial x}\right) \geqslant 0$ and (3.6) shows that $\varphi(t, x) \geqslant \varphi_{0}(x)$. Thus we have proved:

Proposition 3.1. Let $r$ be of class $C^{1,1}$ in an open set containing $\Lambda_{\varphi_{0}}=$ $\left\{\left(x, \frac{2}{i} \frac{\partial \varphi_{0}}{\partial x}(x)\right) ; x \in \Omega\right\}$ where $\Omega$ is open in $C^{n}$ and $\varphi_{0} \in C^{2}(\Omega)$. Let $H \Subset \Lambda_{\varphi_{0}}$ be a barrier. Then there exists $\varepsilon_{0}<0$ such that $\varphi(t, x) \geqslant \varphi_{0}(x)$ for all $(t, x) \in$ $\left[0, \varepsilon_{0}\right] \times \Pi_{x}(H)$. Here $\varphi(t, x)$ is the solution of (3.6).

A local situation that we shall encounter in the next section is the following: Let $\Gamma \subset \Lambda_{\varphi_{0}}$ be an analytic submanifold of codimension $d$, passing through $\left(x_{0}, \xi_{0}\right)$ and given by the equations $f_{1}(x, \xi)=\cdots=f_{d}(x, \xi)=0$, where $f_{j}$ are real (on $\left.\Lambda_{\varphi_{0}}\right)$. Let $W$ be a small complex neighborhood of $\left(x_{0}, \xi_{0}\right)$ and let $r \in C^{1,1}(W)$ be real on $\Lambda_{\varphi_{0}} \cap W$ and holomorphic in $W_{\mathbf{c}}=\{(x, \xi) \in W$; $|\operatorname{Im} \vec{f}(x, \xi)|<\varepsilon|\operatorname{Re} \vec{f}(x, \xi)|\}$ where $\varepsilon>0$, and $\vec{f}=\left(f_{1}, \cdots, f_{d}\right)$. Then (near $\left.\left(x_{0}, \xi_{0}\right)\right)$, $\bar{\partial} r$ vainshes at the points of $\Gamma^{\mathcal{c}}$ and $\frac{\partial r}{\partial x_{j}}, \frac{\partial r}{\partial \xi_{j}}$ are holomorphic on $\Gamma^{c}$. Assume that

$$
H_{r} \text { is tangent to } \Gamma \text {. }
$$

Then we can consider $H_{r}$ as a vector field with holomorphic coefficients of type $(1,0)$ on $\Gamma^{c}$ and for small values of $t$ we have near $\left(x_{0}, \xi_{0}\right)$; $\exp \left(\widehat{i t H_{r}}\right)\left(\Lambda_{\varphi_{0}}\right)$ $\subset W_{\text {e }}$, exp ${\widehat{\left(i t H_{r}\right.}}_{r}(\Gamma) \subset \Gamma^{c}$. This means that we can solve the problem (3.7). If in addition $\Gamma \subset \Sigma \subset \Lambda_{\varphi_{0}}$ with $\Sigma^{c}$ as above, and $H_{r}$ is tangent to $\Sigma$ then $\left.\exp \widehat{\left(i t H_{t}\right.}\right)(\Sigma) \subset \Sigma^{c}$.

We now assume in addition that $\gamma:[-\varepsilon, \varepsilon] \rightarrow \Gamma, \gamma(0)=\left(x_{0}, \xi_{0}\right), \gamma(t)=$ $(x(t), \xi(t))$ is an integral curve of $H_{r}$ on which $r$ vanishes. Then $\left.\Pi_{x}\right|_{r} c$ is a local holomorphic diffeomorphism onto $\Pi_{x}\left(\gamma^{c}\right)$ which is then also a complex curve. If we imbed $\gamma$ in a "real" Lagrangian manifold $\Lambda \subset \Lambda_{\varphi_{0}}$ then $\Lambda^{c}=\Lambda_{\psi}$ where $\phi \leqslant \varphi_{0}$ with equality on $\Pi_{x}(\Lambda) \supset\left(\Pi_{x}(\gamma)\right.$. On $\Pi_{x}\left(\gamma^{\mathcal{C}}\right)$ we have 
$\left(x, \frac{2}{i} \frac{\partial \psi}{\partial x}\right) \in \gamma^{c},\left.\phi\right|_{\Pi_{x}(\gamma)}=\varphi_{0}$ and these properties determine $\phi$ uniquely on $\Pi_{x}\left(\gamma^{c}\right)$. Since $\psi$ is pluri-harmonic, its restriction to $\Pi_{x}\left(\gamma^{\mathcal{C}}\right)$ is harmonic and since $\varphi_{0}$ is strictly plurisubharmonic, we conclude easily that on $\Pi_{x}(\gamma)$ : $\left(\varphi_{0}-\psi\right)(x) \sim d\left(x, \Pi_{x}(\gamma)\right)^{2}$. (See [14] and Lebeau [10]).

Let $\varphi(t, x)$ be the solution of the problem (3.7). Then at the point $(t, x(-i t)) \in \boldsymbol{C} \times \Pi_{x}\left(\gamma^{c}\right)$ we have $\frac{\partial \varphi}{\partial t}=0, \frac{2}{i} \frac{\partial \varphi}{\partial x}=\xi(-i t)=\frac{2}{i} \frac{\partial \psi}{\partial x}(x(-i t))$. Following the complex curve $t \rightarrow(t, x(-i t))$ we find $\varphi(t, x(-i t))=\psi(x(-i t))$ for $t \in \boldsymbol{C},|t| \leqslant \varepsilon$. Since $r$ is real on $\Lambda_{\varphi_{0}}$ and hence on $\Lambda_{\varphi_{t}}$, we have $\varphi(t, x)=$ $\varphi(\operatorname{Re} t, x)$ by $(3.7)$ and hence

$$
\phi(x(s-i t))=\varphi(t, x(s-i t)), \quad(s, t) \in \boldsymbol{R}^{2},|(s, t)| \leqslant \varepsilon .
$$

In this relation $\Pi_{x}\left(\gamma^{c}\right)$ is identified locally with $C$ and $\Pi_{x}(\gamma)$ with the real axis. A trivial consequence is that if $\varepsilon_{0}>0$ is given and if $x \in \Pi_{x}\left(\gamma^{c}\right)$ is sufficiently close to $\Pi_{x}(\gamma)$ and has a positive $t$ coordinate (when writing $x=x(s-i t))$ then

$$
\psi(x) \leqslant \min _{0 \leqslant t \leqslant o_{0}} \varphi(t, x) .
$$

As matter of fact, we even have equality in (3.11).

\section{Applications}

Let $P$ be a differential operator of order $M$ with analytic coefficients defined near a point $x_{0} \in \boldsymbol{R}^{n}$. We shall study $P$ microlocally near a point $\left(x_{0}, \xi_{0}\right) \in T^{*} \boldsymbol{R}^{n} \backslash 0$. Let $\Sigma \subset \boldsymbol{R}^{2 n}$ be an analytic submanifold of codimension $d$, passing through $\left(x_{0}, \xi_{0}\right)$ and let $\Sigma^{c}$ be its complexification. We assume throughout this section that $P$ is of class $S^{M, m}(\Sigma)$ (see [2]), transversally elliptic, and that $P$ satisfies the necessary and sufficient conditions of microlocal hypoellipticity with loss of $m / 2$ derivatives of Boutet de Monvel-GrigisHelffer [2]. Possibly after replacing $P$ by $P * P$ and $(M, m)$ by $(2 M, 2 \mathrm{~m})$, if we put $A\left(x, \tilde{D}_{x}, \lambda\right)=P\left(x, \tilde{D}_{x}, \lambda\right)=\lambda^{-M} P\left(x, D_{x}\right)$ then we are precisely in the situation described in the beginning of section 2 . We shall frequently apply the FBI-transform (2.6) and by abuse of notation we denote the transformed operator still by the letter $P$, the image of $\left(x_{0}, \xi_{0}\right)$ still by $\left(x_{0}, \xi_{0}\right)$ and so on. It will be clear from the context if we work on the transform side or not.

As a first application we give a new proof of the analytic hypoellipticity in the symplectic case. This result was proved for $m=2$ by $\mathrm{F}$. Trèves [19] and D. Tartakoff [17] and later by G. Metivier [11] in the following general form, using a different method. Tartakoff [18] has also extended his methods 
to cover the general case.

TheOREm 4.1. Assume that $\Sigma$ is symplectic. Then if $u$ is a distribution defined near $x_{0}$, such that $\left(x_{0}, \xi_{0}\right) \notin W F_{a}(P u)$, we have $\left(x_{0}, \xi_{0}\right) \notin W F_{a}(u)$.

Proof. Here as well as below we shall proceed by constructing a suitable "weight-function" $r(x, \xi)$ in order to deform $\varphi_{0}$ as in section 3. The mapping $F$ introduced in section 2 , will then be defined as $\exp \left(-t H_{\mathrm{R} e r}^{\operatorname{Im} \sigma}\right)$ or when $r$ is analytic symply as $\exp \left(-i t H_{r}\right)$. When $t$ is small this mapping will be close to the identity in Lipschitz norm. To assume the crucial condition $F\left(\Lambda_{\varphi_{0}} \cap \Sigma^{\mathcal{C}}\right)=\Lambda_{\varphi_{t}} \cap \Sigma^{\mathcal{C}}$ it suffices in the analytic case that $H_{r}$ be tangent to $\Sigma$ or in other words that $\left.d r\right|_{T^{\perp}}=0$ at every point of $\Sigma$. Here $T \Sigma^{\perp}$ is the orthogonal space of $T \Sigma$ with respect to the symplectic form. If $\Sigma$ is given by the equations $f_{1}=\cdots=f_{d}=0$ where $f_{1}, \cdots, f_{d}$ are analytic and real on $\boldsymbol{R}^{2 n}$ (that we identify with $\Lambda_{\varphi_{0}}$ by using $\mathscr{A}_{T}$ ), then we look for analytic functions $r$ such that

$$
H_{f_{j}}(r)=0 \text { on } \Sigma \text { for } j=1, \cdots, d .
$$

When $\Sigma$ is symplectic, $T \Sigma^{\perp}$ is transversal to $T \Sigma$ everywhere and we can prescribe any analytic function $\rho$ on $\Sigma$ and construct locally and analytic solution $r$ to the problem (4.1) such that $\left.r\right|_{z}=\rho$. Also if $r$ is a solution of (4.1) we can make it more positive outside $\Sigma$ by adding $R \sum_{1}^{d} f_{j}^{2}$ where $R \gg 0$. In the symplectic case it is easy to see we have an analytic solution $r$ to the problem (4.1) in some neighborhood of $\left(x_{0}, \xi_{0}\right)$ such that $\left.r\right|_{R^{2 n}} \sim\left|\dot{x}-x_{0}\right|^{2}+$ $\left|\xi-\xi_{0}\right|^{2}$. After the FBI transform we have $r\left(x, \frac{2}{i} \frac{\partial \varphi_{0}}{\partial x}\right) \sim\left|x-x_{0}\right|^{2}$ and if we fix small open sets $\Omega \supset \Omega_{1} \supset \Omega_{2} \ni x_{0}$ we can apply Theorem 2.1 with $\varphi=\varphi_{t}$ where $\varphi_{t}(x)=\varphi(t, x)$ is the solution of (3.6) (or (3.7)). Denoting by $u$ also the transformed holomorphic function and $P$ a suitable realisation, as in section 2, of the transformed operator, we have $\|u\|_{\varphi_{0}, 2}=\mathscr{O}(1) \lambda^{N_{0}},\|P u\|_{\varphi_{0}, a_{3}}=$ $\mathcal{O}(1) e^{-\lambda / C}, C>0$ if $\Omega_{3}$ is a sufficiently small neighborhood of $x_{0}$. Since $\varphi_{t}-\varphi_{0} \sim$ $t\left|x-x_{0}\right|^{2}$ we get $\|P u\|_{\varphi_{t}, \Omega_{1}}=\mathscr{O}(1) e^{-\lambda / C_{t}}, C_{t}>0$ and also $\|u\|_{\varphi_{t}, \Omega \backslash \Omega_{2}}=\mathscr{O}(1) e^{-\lambda / C_{t}}$. If $t>0$ is small enough we can apply Theorem 2.1 and get $\|u\|_{i_{\varphi_{t}}, a_{2}}=$ $\mathscr{O}(1) e^{-\lambda / C_{t}}$ and hence that $\| u_{\varphi_{0}, a_{4}}=\mathscr{O}(1) e^{-\lambda / 2 C_{t}}$ if $\Omega_{4}$ is a small neighborhood $x_{0}$, where $\varphi_{t} \leqslant \varphi_{0}+1 / 3 C_{t}$. Before the FBI transform we than have $\left(x_{0}, \xi_{0}\right) \notin$ $W F_{a}(u)$. The proof is complete.

The new results of this paper are rather special and concern mostly the case when $\Sigma$ is of codimension 2 . We extend them later to the case when $\Sigma$ is of higher codimension but $\operatorname{dim} T \Sigma \cap T \Sigma^{\perp} \leqslant 2$. Thus let us assume that $\Sigma$ is given by the two real equations $f_{1}(x, \xi)=f_{2}(x, \xi)=0 . \quad \Sigma$ is then 
non-symplectic precisely at the points where $g=\left\{f_{1}, f_{2}\right\}$ vanishes. If we assume first that $g$ (defined on $\Sigma$ ) vanishes on a hypersurface $\Gamma \subset \Sigma$ passing through $\left(x_{0}, \xi_{0}\right)$, then the "generic" situation is that

$$
T \Gamma+\left(T \Sigma^{\perp} \cap T \Sigma\right)=T \Sigma \quad \text { at every point of } \Gamma .
$$

In the case when $\Gamma$ contains an integral manifold of $T \Sigma^{\perp} \cap T \Sigma$ it has been proved in Grigis-Schapira-Sjöstrand [3] ([14]) that analytic singularities of solutions to the equation $P u=v$ propagate along this integral manifold in the complement of $W F_{a}(v)$. That singular solutions exist was proved by Metivier [12] when $m=2$.

Under the assumption (4.2) $T \Gamma \cap T \Sigma^{\perp}$ is of dimension 1 at every point and we can choose $f_{1}, f_{2}$ so that this space is generated by $H_{f_{1}}$ at every point of $\Gamma$, while $H_{f_{2}}$ is transversal to $\Gamma$. If we represent $\Gamma$ in $\Sigma$ by an equation $h=0$ where $h$ is real, analytic and $d h \neq 0$ then our last assumption is

There exists an integer $\mathscr{H} \geqslant 1$ such that $g / h^{\mathscr{*}}$ is a nonvanishing analytic function in a neighborhood of $\left(x_{0}, \xi_{0}\right)$ in $\Sigma$.

Let $\left.\gamma_{0}:\right]-\varepsilon, \varepsilon\left[\rightarrow \Gamma\right.$ be a segment of the integral curve of $H_{f_{1}}$ with $\gamma_{0}(0)=$ $\left(x_{0}, \xi_{0}\right)$.

THEOREM 4.2. Let us assume that $\Gamma \subset \Sigma$ is an analytic hypersurface containing $\left(x_{0}, \xi_{0}\right)$ and that (4.2), (4.3) hold. Define $\gamma_{0}$ as above, (as an integral curve of $\left.T \Gamma \cap T \Sigma^{\perp}\right)$. If $u \in \mathscr{D}^{\prime}\left(\boldsymbol{R}^{n}\right)$ and $\gamma_{0} \cap W F_{a}(P u)=\phi$, then either $\gamma_{0} \subset W F_{a}(u)$ or $\gamma_{0} \cap W F_{a}(u)=\phi$.

PROof. The geometric discussion that follows will be local in the real domain. We can choose real symplectic corrdinates near $\left(x_{0}, \xi_{0}\right) \in \Gamma$ such that $f_{1}=\xi_{n}$ and $f_{2}=\left(\xi_{n-1}+r\left(x, \xi^{\prime}, \xi_{n}\right)\right)$ times an elliptic factor. Dividing out the elliptic factor and modifying $f_{2}$ in the region where $f_{1} \neq 0$ we can assume $f_{2}=\xi_{n-1}+r\left(x, \xi^{\prime}\right)$. Here $\xi^{\prime}=\left(\xi_{1}, \cdots, \xi_{n-2}\right)$. We still have $H_{f_{2}} \notin T \Gamma$. The new function $g\left(x, \xi^{\prime}\right)=\left\{f_{1}, f_{2}\right\}$, differs from the old one on $\Sigma$ only by an elliptic factor, and is independent of $\xi_{n}, \xi_{n-1}$. We can write $g=h\left(x, \xi^{\prime}\right)^{2}$ where $\Gamma=H \cap \Sigma: f_{1}=f_{2}=h=0$ if we define $H$ to be the hypersurface given by $h\left(x, \xi^{\prime}\right)=0$. Since $H_{f_{1}}$ is tangent to $\Gamma$ and $h$ and $H_{f_{1}}$ are invariant under translations in the $\left(\xi_{n-1}, \xi_{n}\right)$ directions we see that $H_{f_{1}}$ is tangent to $H$. Let $\rho^{0}$ be a real function on $\Gamma$ satisfying the equation $H_{f_{1}}\left(\rho^{0}\right)=0$. Let $G^{\prime} \subset \Gamma$ be a hypersurface transversal to $H_{f_{1}}$ and let $G \subset H$ be a hypersurface with $G \cap \Gamma=\Gamma^{\prime}$, transverse to $H_{f_{1}}$ and invariant under the $H_{h}$ flow. $\left(H_{h}\right.$ is nontangent to $\Gamma$ since $\left.H_{h}\left(f_{2}\right) \neq 0\right)$.

We can extend $\left.\rho^{(0)}\right|_{a^{\prime}}$ to $G$ in a non-unique way such that $H_{h}\left(\rho^{(0)}\right)=0$ on $G$. We then extend this function to a function $\rho^{(1)}$ on $H$ such that 
$H_{f_{1}}\left(\rho^{(1)}\right)=0$. Then $\left.\rho^{(1)}\right|_{\Gamma}=\rho^{(0)}$. Since $\left\{f_{1}, h\right\}=\mathscr{O}(h)$ we have $\left[H_{f_{1}}, H_{h}\right]=$ $a(x, \xi) H_{h}$ on $H$, hence $H_{f_{1}}\left(H_{h}\left(\rho^{(1)}\right)\right)=a\left(H_{h}\left(\rho^{(1)}\right)\right)$ and $H_{h}\left(\rho^{(1)}\right)$ vanishes on $H$.

We choose local coordinates, centered at $\left(x_{0}, \xi_{0}\right)$, in the following way: First let $z^{\prime}$ be local coordinates on $G^{\prime}$. Then let $\left(z^{\prime}, t^{\prime}, t^{\prime \prime}\right)$ be local coordinates on $G$ such that $H_{h}=\frac{\partial}{\partial t^{\prime}}$ on $G$ and $t=\left(t^{\prime}, t^{\prime \prime}\right)=0$ on $G^{\prime}$. Extend these coordinates to $H$ and add one; $z^{\prime \prime}$ so that $G$ is given by $z^{\prime \prime}=0$ and $H_{f_{1}}=$ $\frac{\partial}{\partial z^{\prime \prime}}$ on $H$. Then $t=\left(t^{\prime}, t^{\prime \prime}\right)$ vanishes on $\Gamma$ and the preceding remark about the commutator of $H_{f_{1}}$ and $H_{h}$ shows that $H_{h}=b \frac{\partial}{\partial t^{\prime}}$ on $H$, where $b$ is a non-vanishing function. Finally extend the coordinates $(z, t)$ to a full neighborhood of $\left(x_{0}, \xi_{0}\right)$ and add one coordinate $s$, in such a way that $s=0$ on $H$ and $H_{f_{2}}=\frac{\partial}{\partial s}$. Clearly $s$ and $h$ differ only by an elliptic factor.

We can choose $\rho^{(1)}$ above so that $\rho^{(1)}(z, t)=\rho^{(0)}\left(z^{\prime}\right)$. If $k(z)$ is an analytic function on $\Gamma$, to be choosen later, we put

$$
\rho(z, t, s)=\rho^{(1)}(z, t)+k(z) \frac{s^{z+1}}{\mathscr{A}+1} .
$$

Then

$$
H_{f_{2}}(\rho)=k(z) s^{*}
$$

We have

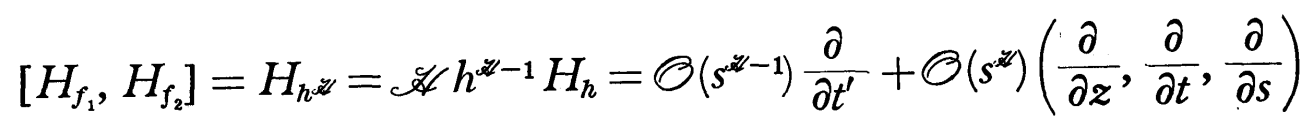

so that

$$
H_{f_{1}}=\frac{\partial}{\partial z^{\prime \prime}}+\mathscr{O}\left(s^{*}\right) \frac{\partial}{\partial t^{\prime}}+\mathscr{O}\left(s^{*+1}\right)\left(\frac{\partial}{\partial z}, \frac{\partial}{\partial t}, \frac{\partial}{\partial s}\right) .
$$

It follows that

$$
H_{f_{1}}(\boldsymbol{\rho})=\Theta\left(s^{z+1}\right) \text {. }
$$

If $R>0$ is a sufficiently large constant we put

$$
r=\rho-\frac{H_{f_{1}}(\rho)}{g} f_{2}+\frac{H_{f_{2}}(\rho)}{g} f_{1}+R\left(f_{1}^{2}+f_{2}^{2}\right)
$$

which is a real and analytic function satisfying (4.1).

The bicharacteristic segment $\gamma_{0}$ is given in $\Gamma$ by the equations $z^{\prime}=0$.

We now choose $\rho^{(0)}=\left|z^{\prime}\right|^{2}$. Then 


$$
H_{r}=\left(\frac{s}{h}\right)^{2} k(z) H_{f_{1}} \in T \gamma_{0} \quad \text { on } \quad \gamma_{0} .
$$

Let $A$ be a small sphere in $\Gamma$ (for the coordinates $z$ ) centered at 0 . We shall choose $k$ vanishing to the second order on $A$. Then the cylindrical hypersurface $C=\{(z, t, s) ; z \in A\}$ is a barrier for the weight function (4.7). In fact, on $C$ we have $H_{f_{1}}(\rho)=\mathscr{O}\left(s^{z+1}\left|z^{\prime}\right|\right)$ so

$$
\frac{H_{f_{1}}(\rho)}{g} f_{2}=\mathscr{O}\left(s\left|z^{\prime}\right| f_{2}\right)=\mathscr{O}\left(s\left(\left|z^{\prime}\right|^{2}+f_{2}^{2}\right)\right) \text {. }
$$

Moreover $H_{f_{2}}(\rho)=0$ on $C$, so $r \geqslant 0$ is of the same order of magnitude as $\left|z^{\prime}\right|^{2}+f_{1}^{2}+f_{2}^{2}$ on $C$. Since the gradient of each term in (4.7) can be estimated on $C$ by a constant times $\left|\boldsymbol{z}^{\prime}\right|+\left|f_{1}\right|+\left|f_{2}\right|$ our claim follows.

We now assume that we have worked in a small neighborhood of $\left(x_{0}, \xi_{0}\right)$ whose closure is disjoint from $W F_{a}(P u)$. Then we perform our FBI-transform and let $u$ be the transformed function while $(z, t, s)$ are considered as coordinates on $\Lambda_{\varphi_{0}}$ or even on $\boldsymbol{C}_{x}^{n}$. Let then $\Omega_{0} \subset \boldsymbol{C}_{x}^{n}$ be given by $\boldsymbol{z} \in B$, $|t|<\alpha,|s|<\alpha$ where $\alpha>0$ is small and fixed $>0, B$ is the open ball with border $A$. Since we already know that $P$ is a.h.e. outside $\Gamma$ we know that there is a function $\tilde{\psi}$ of class $C^{2}$ in a neighborhood of $\bar{\Omega}_{0}$ such that $u \in H_{\tilde{\beta}}^{\text {loc }}$ and $\tilde{\psi}=\varphi_{0}$ on $\Pi_{x}(\Gamma): t=0, s=0$ while $\tilde{\phi}<\varphi_{0}$ elsewhere. Moreover in some smaller neighborhood of $\bar{\Omega}_{0}$, we have $P u \sim 0$ in $H_{\tilde{\psi}}^{\text {loc }}$ if $P$ denotes a realisation of the transformed operator with a regular contour adapted to $\varphi_{0}$ as in (2.16). A boundary point of $\Omega_{0}$ is either in the barrier $C$, or else $|t|$ or $|s|$ is equal to $\alpha$. Hence for $0 \leqslant t \leqslant \varepsilon_{0}>0$ small enough we have $\varphi_{t} \geqslant \tilde{\psi}$ on $\partial \Omega_{0}$. Let

$$
\begin{aligned}
P_{\Omega_{0}} u & =\left(\frac{\lambda}{2 \pi}\right)^{n} \iint e^{i \lambda(x-y) \xi} P(x, \xi) \chi(x, y) u(y) d y d s \\
\xi & =\frac{2}{i} \frac{\partial \tilde{\psi}}{\partial x}(x)+\frac{i}{C} \frac{(\overline{x-y})}{|x-y|}
\end{aligned}
$$

be a realisation of $P$ in $\Omega_{0}$ where $\chi \in C^{\infty}\left(\Omega_{0} \times \Omega_{0}\right)$ is equal to 1 and has its support in two sets of the form $\left\{(x, y) \in \Omega_{0} ;|x-y| \leqslant \frac{1}{\text { const. }} d\left(x, C \Omega_{0}\right)\right\}$. Then as we saw in [14], section 12 , we have

$$
P u-P_{\Omega_{0}} u \in L_{\hat{\psi}}^{2,1 \text { oc }}\left(\Omega_{0}\right), \quad \hat{\psi}(x)=\tilde{\psi}(x)-\frac{1}{\tilde{C}} d\left(x, \complement \Omega_{0}\right) .
$$

It $\varepsilon_{0}>0$ is small enough it follows that

$$
P_{\Omega_{0}} u \in L_{\varphi_{t}}^{2,100}\left(\Omega_{0}\right), \quad 0 \leqslant t \leqslant \varepsilon_{0} .
$$


For $\tau>0$ define $\Omega, \Omega_{1}, \Omega_{2}$ to be the open subsets of $\Omega_{0}$, where respectively $d\left(x, \mathcal{G} \Omega_{0}\right)>\tau, 2 \tau, 3 \tau$. If $\varepsilon_{0}>0$ is small enough, but independent of $\tau$, we can apply Theorem 2.1, with $P=P_{s}$ :

$$
\|u\|_{\varphi_{t}, \Omega_{2}} \leqslant C_{\tau}\left(\left\|P_{o} u\right\|_{\varphi_{t}, \Omega_{1}}+\|\| u \|_{\varphi_{t}, \Omega \backslash \Omega_{2}}\right) \text {. }
$$

Here $\left\|P_{g} u\right\|_{\varphi_{t}, a_{1}}$ is at most of polynomial increase in $\lambda$ as $\lambda \rightarrow \infty$, while $u \|_{\varphi_{t}, \Omega \backslash \Omega_{2}} \leqslant C_{\tau} e^{\tau_{\tau} \lambda}$, since $\varphi_{t} \geqslant \psi(x)-$ (const.) $\tau$ in $\Omega \backslash \Omega_{2}$. Letting $\tau$ tend to 0 we conclude that

$$
u \in H_{\varphi_{t}}^{\text {loc }}\left(\Omega_{0}\right), \quad 0 \leqslant t \leqslant \varepsilon_{0} .
$$

If we choose first $k>0$, then $K<0$ on $\gamma_{0} \cap B$, we conclude from the disscussion in the end of section 3 , that $u \in H_{\Phi}^{\text {loc }}\left(\Omega_{0}\right)$ where $\Phi$ is a Lipschitz function $\leqslant \psi$ on a neighborhood of $\Pi_{x}\left(\gamma_{0}\right) \cap B$ in $\Pi_{x}\left(\gamma_{0}^{c}\right)$. Here $\phi$ is the harmonic function introduced in the end of section 3 (where the bicaracteristic segment was called $\gamma$ ). Using the maximum principle it follows that if $u \sim 0$ in $H_{\varphi_{0}}^{\text {loc }}$ at a point of $\Pi_{x}(\gamma) \cap B$, then the same is true at every point in this set. Translated into the original terms before the FBI-transform, this means that either $\gamma_{0} \subset B$ is disjoint from, or contained in, the analytic wave front set of $u$. Since $\gamma_{0}$ can be covered by a finite number of balls of the type $B$, the Theorem follows.

It should be pointed out that we don't know that the operator in Theorem 4.2 is not analytic hypoelliptic at $\left(x_{0}, \xi_{0}\right)$. Such hypoellipticity would of course then be a stronger result than Theorem 4.2. The same uncertainty affects some of the results below.

We still assume that $\Sigma: f_{1}=f_{2}=0, g=\left\{f_{1}, f_{2}\right\}$. Let $\Gamma \subset \Sigma$ be an analytic submanifold passing through $\left(x_{0}, \xi_{0}\right)$ and assume that there is an even integer $\mathscr{A}>0$ such that

$$
g \sim d_{\Gamma}^{\ddot{y}} \text { on } \Sigma,
$$

where $d_{\Gamma}$ denotes the distance to $\Gamma$. When the codimension of $\Gamma$ in $\Sigma$ is larger than or equal to 2 , then the "generic" situation is that

$$
\left(T \Sigma \cap T \Sigma^{\perp}\right) \cap T \Gamma=0
$$

at every point of $\Gamma$. Here $T \Sigma \cap T \Sigma^{\perp}$ is the space spanned by $H_{f_{1}}, H_{f_{2}}$ (at the points of $\Gamma$ ). The last assumption is that

$$
\left.\sigma\right|_{\Gamma} \text { is of constant rank on } \Gamma \text {. }
$$

Then $\Gamma$ has a bicharacteristic foliation of submanifolds whose tangent space at every point is $T \Gamma \cap T \Gamma^{\perp}$. When $\Gamma$ is not symplectic, let $L$ be such a 
connected integral manifold containing $\left(x_{0}, \xi_{0}\right)$, and contained in the small real neighborhood of $\left(x_{0}, \xi_{0}\right)$ where the above assumptions are made.

TheOREM 4.3. We assume (4.13)-(4.15). $W F_{a}(u)$.

(a) If $\Gamma$ is symplectic, $u \in \mathscr{D}^{\prime}\left(\boldsymbol{R}^{n}\right),\left(x_{0}, \xi_{0}\right) \notin W F_{a}(P u)$, then $\left(x_{0}, \xi_{0}\right) \notin$

(b) If $\Gamma$ is non symplectic, $L$ as above, and $u \in \mathscr{D}^{\prime}\left(\boldsymbol{R}^{n}\right)$ satisfies $L \cap$ $W F_{a}(P u)=\phi$, then either $L \subset W F_{a}(u)$ or $L \cap W F_{a}(u)=\phi$.

PRoof. In a sufficiently small neighborhood we shall construct a weight of class $C^{1,1}$. Let

$$
T \Gamma^{\perp}=\mathscr{T}_{1} \oplus \mathscr{T}_{2} \oplus\left(H_{f_{1}}, H_{f_{2}}\right)
$$

be a non-unique decomposition in the sense of vector bundles, where $\mathscr{T}_{1}=$ $T \Gamma \cap T \Gamma^{\perp}$. Let $H \subset \boldsymbol{R}^{2 n}$ be of codimension 2 and such that $\Gamma \subset H, \mathscr{T}_{1} \oplus \mathscr{T}_{2}$ $\left.\subset T H\right|_{\Gamma}$ and $\left(H_{f_{1}}, H_{f_{2}}\right)$ is transversal to $T H$. Since $\mathscr{T}_{1}$ is the tangent space of $L$ at $\left(x_{0}, \xi_{0}\right)$ we see that

$$
\operatorname{dim} H=\operatorname{dim} \Gamma+\operatorname{dim} \mathscr{T}_{2}+\operatorname{dim} L .
$$

We choose local coordinates centered at $\left(x_{0}, \xi_{0}\right)$ in the following way. On $\Gamma$ we take $z=\left(z^{\prime}, z^{\prime \prime}\right)$ in such a way that $\mathscr{T}_{1}$ at every point is spanned by the $\frac{\partial}{\partial z^{\prime \prime}}$ directions. On $H$ we extend the coordinates $z$ and add the coordinates $t=\left(t^{\prime}, t^{\prime \prime}\right)$ in such a way that $t=0$ on $\Gamma$ and $\mathscr{T}_{2}$ at every point of $\Gamma$ is spanned by the $\frac{\partial}{\partial t^{\prime}}$-directions. By (4.17), we have as many $z^{\prime \prime}$ coordinates as $t^{\prime \prime}$ coordinates. After a preliminary reduction to $f_{1}=\xi_{n}, f_{2}=\xi_{n-1}+r\left(x, \xi^{\prime}\right)$, which implies that $g=\mathscr{O}\left(d_{\Gamma}^{*}\right)$ in a full neighborhood of $\left(x_{0}, \xi_{0}\right)$, we extend the coordinates $(z, t)$ to a full neighborhood of $\left(x_{0}, \xi_{0}\right)$ and complete by $s=\left(\mathrm{s}_{1}, s_{2}\right)$ in such a way that $s=0$ on $H, H_{f_{1}}=\frac{\partial}{\partial s_{1}}$ and $s_{2}=0$ on $\mathscr{H}=\exp \left(\boldsymbol{R} H_{f_{1}}\right)(H)$ and $H_{f_{2}}=\frac{\partial}{\partial s_{2}}$ everywhere. Since $g=\mathscr{O}\left(d_{\Gamma}^{*}\right)$ and $T \Gamma^{\perp}$ is spanned by the directions $\frac{\partial}{\partial z^{\prime \prime}}, \frac{\partial}{\partial t^{\prime}}, \frac{\partial}{\partial s}$, we have

$$
\begin{gathered}
{\left[H_{f_{1}}, H_{f_{2}}\right]=H_{g}=\mathscr{O}\left((t, s)^{z-1}\right)\left(\frac{\partial}{\partial z^{\prime \prime}}, \frac{\partial}{\partial t^{\prime}}, \frac{\partial}{\partial s}\right)} \\
+\mathscr{O}\left((t, s)^{z^{z}}\right)\left(\frac{\partial}{\partial z}, \frac{\partial}{\partial t}, \frac{\partial}{\partial s}\right)
\end{gathered}
$$

in the sense that the coefficients of $\frac{\partial}{\partial z^{\prime \prime}}, \frac{\partial}{\partial t^{\prime}}, \frac{\partial}{\partial s}$ are analytic functions which are $\mathscr{O}\left((t, s)^{*-1}\right)$ and so on. 
Let $\rho^{(0)}(z)=\rho^{(0)}\left(z^{\prime}\right)$ be a real and analytic function on $\Gamma$ with $\left.d \rho^{0}\right|_{T \Gamma \cap T \Gamma^{\perp}}$ $=0$ at every point. Extend $\rho^{(0)}$ to a function $\rho^{(1)}(z, t)$ in such a way that $\left.d \rho^{(1)}\right|_{\mathscr{T}_{1} \oplus \mathcal{F}_{2}}=0$ at every point of $\Gamma$. For instance we can take

$$
\rho^{(1)}=\rho^{(0)}\left(z^{\prime}\right)+\vec{b}(z) \cdot t^{\prime \prime}
$$

where $\vec{b}$ is a real and analytic vector. (The most general choice would be to add an arbitrary term $\mathscr{O}\left(t^{2}\right)$ in $\left.(4.19)\right)$. Finally we put

$$
\rho(z, t, s)=\rho^{(1)}(z, t)=\rho^{(1)}\left(z, t^{\prime \prime}\right) .
$$

Then

$$
\left.d \rho\right|_{T \Gamma^{\perp}}=0 \text { everywhere on } \Gamma,
$$

$$
H_{f_{2}}(\rho)=0 \text { everywhere, }
$$

and $H_{f_{1}}(\rho)=0$ on $\mathscr{A}$ so that at an arbitrary point:

$$
H_{f_{1}}(\rho)(z, t, s)=\int_{0}^{s_{2}} H_{g}(\rho)\left(z, t, s_{1}, \sigma\right) d \sigma .
$$

In view of (4.18), (4.19) we obtain $H_{g}(\rho)=\mathscr{O}\left((t, s)^{z^{*}} \nabla b\right)+\mathscr{O}\left((t, s)^{z^{2}} \nabla \rho^{(0)}\right)+$ $\mathcal{O}\left((t, s)^{2} \vec{b}\right)$

$$
H_{f_{1}}(\rho)(z, t, s)=\mathscr{O}\left((t, s)^{z+1}\right) \nabla \rho^{(0)}+\mathscr{O}\left((t, s)^{z+1}\right) \nabla \vec{b}+\mathscr{O}\left((t, s)^{z+1} \vec{b}\right) .
$$

Let $\tilde{g}$ be an analytic and real extension of $\left.g\right|_{\Sigma}$ such that $\tilde{g} \sim d_{\Gamma}^{*}$ and put

$$
r=\rho-\frac{H_{f_{1}}(\rho)}{\tilde{g}} f_{2}+f_{2}^{2}
$$

Then $r$ is of class $C^{1,1}$ in the real domain and has a holomorphic extension to domain of the form $|z|<\varepsilon,|\operatorname{Im}(t, s)|<\varepsilon|\operatorname{Re}(t, s)|<\varepsilon^{2}$. Introducting a cut off function $\chi\left(|\operatorname{Re}(t, s)|^{-1} \operatorname{Im}(t, s)\right)$ in front of the middle term in (4.24), where $\chi \in C_{0}^{\infty}$ is equal to 1 near 0 and has a sufficiently small support, we achieve that $r$ is of class $C^{1,1}$ in a full complex neighborhood of $\left(x_{0}, \xi_{0}\right)$, while still holomorphic and of the form (4.24) in a (somewhat smaller) complex domain of the type above. $r-\rho$ vanishes to the second order on $\Gamma$ so (4.21) shows that

$$
H_{r} \in T \Gamma \text { everywhere on } \Gamma \text {. }
$$

(Also, $H_{r}$ is tangent to $\Sigma$ by construction).

In the case (a) the proof is now easily concluded. We choose $\rho^{(0)}=$ $|z|^{2}-\delta^{2}$ where $\delta>0$ is small enough. The set $C=\{(z, t, s) ;|z|=2 \delta,|(t, s)| \leqslant \delta\}$ is easily seen to be a barrier simply because $r>0$ on $C$. Applying our FBItransform, the general remarks in the end of section 3, Theorem 2. 1 as in 
the end of the proof of Theorem 4.2, we see that the transformed function $u$ is of class $H_{\varphi_{t^{\prime}}}^{\text {loc }}$ in the domain $|z|<2 \delta,|(t, s)|<\delta$ when $t^{\prime}>0$ is small enough. Here $\varphi_{t^{\prime}}(x)=\varphi\left(t^{\prime}, x\right)$ solves the problem (3.7), and we have $\varphi_{t^{\prime}}\left(x_{0}\right)<\varphi_{0}\left(x_{0}\right)$ when $t^{\prime}>0$ is small enough, so we conclude that $\left(x_{0}, \xi_{0}\right) \notin W F_{a}(u)$ for the untransformed function. This completes the proof in the case (a).

In the case $(b)$ we recall that $L$ is given by the equations $z^{\prime}=0$ in $\Gamma$. We choose $\rho^{(0)}=\left|z^{\prime}\right|^{2}$. Then on $L$ we have $\left.d r\right|_{r \Gamma}=0$ so $H_{r} \in T \Gamma^{\perp}$. With (4.25) this gives

$$
H_{r} \in T L \text { everywhere on } L \text { and }\left.r\right|_{L}=0 .
$$

We have $\operatorname{dim} L$ degrees of freedom in the choice of $\vec{b}$ so $r$ can be constructed so that $H_{r}$ is any analytic section in $T L$.

Let $B \subset \Gamma$ be the ball $|\boldsymbol{z}|<\boldsymbol{\delta}_{0}$ for some sufficiently small $\delta_{0}>0$ and let $A$ be its boundary in $\Gamma$. We choose $\vec{b}$ to vanish to the second order on $A$ and we shall next verify that for $\delta_{1}>0$ small enough, $C=\{(z, t, s) ; z \in A$, $\left.|(t, s)| \leqslant \delta_{1}\right\}$ is a barrier. In fact, by (4.23), (4.24) we have

$$
r=\left|z^{\prime}\right|^{2}+\Theta\left(\delta_{1}\left|z^{\prime}\right| \cdot\left|f_{2}\right|\right)+f_{2}^{2} \sim\left|z^{\prime}\right|^{2}+f_{2}^{2}
$$

on $C$, while $\nabla r=\mathscr{O}(1)\left(\left|\boldsymbol{z}^{\prime}\right|+\delta_{1}\left|\boldsymbol{z}^{\prime}\right|+\left|f_{2}\right|\right) \leqslant \mathscr{O}(1) r^{1 / 2}$ on $C$. If $\gamma_{0} \Subset B$ is an analytic curve segment in $B$, we choose $r$ so that $H_{r} \neq 0$ on $\gamma_{0}$ and tangent to $\gamma_{0}$. Then by the same arguments as in the proof of Theorem 4.2 we get that if $\bar{B} \cap W F_{a}(P u)=\phi$ then either $\gamma_{0} \cap W F_{a}(u)=\phi$ or $\gamma_{0} \subset W F_{a}(u)$. By varying $\gamma_{0}$ we obtain that either $B \cap W F_{a}(u)=\phi$ or $B \subset W F_{a}(u)$. Covering $L$ by sets of the type $B$, we obtain the statement in $(b)$ of the Theorem. The proof is complete.

In the case $(b)$ of Theorem 4.3 , we cannot prove analytic hypoellipticity using the methods of this section (and the author believes that there is no such regularity result). Indeed, if $r$ is a $C^{1,1}$ function, of class $C^{2}$ a.e. on $\Sigma$, defined in a real neighborhood of $\left(x_{0}, \xi_{0}\right)$, such that $H_{f_{1}}(r)=H_{f_{2}}(r)=0$ on $\Sigma$, then $r$ is constant on each bicaracteristic leaf of $\Gamma$. To see this we first notice that $\left|H_{f_{1}}\right| H_{f_{2}}(r),\left|H_{f_{2}}\right| H_{f_{1}}(r)=\mathscr{O}(g)$ on $\Sigma$, if

$$
\left|H_{f_{1}}\right| v=\varlimsup_{\varepsilon \rightarrow 0} \frac{1}{\varepsilon}\left|v \circ \exp \left(-\varepsilon H_{f_{1}}\right)-v\right| \text {. }
$$

Hence $H_{g}(r)=\mathscr{O}(g)$ on $\Sigma$, since $H_{g}=\left[H_{f_{1}}, H_{f_{2}}\right]$. Let $u_{1}, \cdots, u_{2 n}$ be coordinates centered at $\left(x_{0}, \xi_{0}\right)$ such that $\Gamma$ is given by $u_{1}=\cdots=u_{d+2}=0, u_{d+1}=f_{1}, u_{d+2}=f_{2}$. If is enough to show that $H_{u^{\prime}}(r)=\left(H_{u_{1}}(r), \cdots, H_{u_{d}}(r)\right)=(0, \cdots, 0)$ on $\Gamma$. For simplicity we may do it only at 0 . On $\Sigma$ we have

$$
H_{g}(r)=\sum_{1}^{2 n} \frac{\partial g}{\partial u_{j}} H_{j}(r)=\sum_{1}^{d} \frac{\partial g}{\partial u_{j}} H_{u_{j}}(r)+\mathscr{O}\left(\left|u^{\prime}\right|^{z^{\prime}}\right) \text {, }
$$


since $H_{u_{d+1}}(r)=H_{u_{d+2}}(r)=0$ and $\frac{\partial g}{\partial u_{j}}=\mathscr{O}\left(\left|u^{\prime}\right|^{2}\right)$ for $j \geqslant d+3$. Thus on $\Sigma$ :

$$
\sum_{1}^{d} \frac{\partial g}{\partial u_{j}} H_{u_{j}}(r)=\mathscr{O}\left(\left|u^{\prime}\right|^{2 x}\right)
$$

Put $u_{d+3}=\cdots=u_{2 n}=0$. By Taylors formula and the Euler identity we have

$$
\left|\sum_{1}^{d} \frac{\partial g}{\partial u_{j}} u_{j}\right| \sim\left|u^{\prime}\right|^{2}
$$

We choose $u^{\prime}=\left(u_{1}, \cdots, u_{d}\right)=\varepsilon H_{u^{\prime}}(r)(0)$. Then the above estimate gives

$$
\varepsilon^{-1}\left|H_{u^{\prime}}(r)(0)\right|^{z \prime}=\mathscr{O}\left(\varepsilon^{z^{\prime}}\left|H_{u^{\prime}}(r)(0)\right|^{2}\right)
$$

which implies $H_{u^{\prime}}(r)(0)=0$.

We next look at a complementary case to the two preceeding theorems. We still assume that $\Sigma$ is of codimension 2 , that $\mathscr{A}$ is even $>0$ and that (4.13), (4.15) are valid, where $\Gamma$ is an analytic submanifold. Thinking in particular on the case when $\Gamma$ is a hypersurface we replace $(4.14)$ by the assumption that

$$
\operatorname{dim} T \Gamma \cap T \Sigma^{\perp}=1
$$

at every point of $\Gamma$. We may then recombine $f_{1}, f_{2}$ so that $T \Gamma \cap T \Sigma^{\perp}=\left(H_{f_{1}}\right)$ while $H_{f_{2}}$ is transversal to $T \Gamma$. Let $\gamma_{0}$ be a segment of the integral curve of $H_{f_{1}}$, passing through $\left(x_{0}, \xi_{0}\right)$. The following theorem gives a weaker statement than part $(b)$ of Theorem 4. 3 , and it is perfectly legitimate to wonder if it can be stengthened.

THEOREM 4.4. Under the assumptions (4.13), (4.27), (4.15) with $\mathscr{A}$ even, if $u \in \mathscr{D}^{\prime}\left(\boldsymbol{R}^{n}\right), \gamma_{0} \cap W F_{a}(P u)=\phi$ then either $\gamma_{0} \subset W F_{a}(u)$ or $\gamma_{0} \cap W F_{a}(u)=\phi$.

Proof. Let $H$ be an $H_{f_{1}}$ invariant hypersurface containing $\Gamma$ and transversal to $H_{f_{2}}$. Then we have a fiberbundle decomposition; $T H \cap T \Gamma^{\perp}$ $=\mathscr{T}_{1} \oplus \mathscr{T}_{2}$, where $\mathscr{T}_{1}=T \Gamma \cap T \Gamma^{\perp}$. Then clearly $T \Gamma^{\perp}=\mathscr{T}_{1} \oplus \mathscr{T}_{2} \oplus H_{f_{2}}$. Let $G^{\prime} \subset \Gamma$ be a hypersurface transversal to $H_{f_{1}}$ and $G \subset H$ a hypersurface transversal to $H_{f_{1}}$ containing $G^{\prime}$ and such that $T G \supset \mathscr{T}_{2}$ at every point of $G^{\prime}$. Let $\mathscr{T}_{1}^{\prime}=\mathscr{T}_{1} \cap T G^{\prime}$ so that $\mathscr{T}_{1}=\mathscr{T}_{1}^{\prime} \oplus\left(H_{f_{1}}\right)$ at every point of $G^{\prime}$. We choose local coordinates centered at $\left(x_{0}, \xi_{0}\right)$ in the following way: Let $\left(z^{\prime}, z^{\prime \prime}\right)$ be coordinates on $G^{\prime}$ such that $\mathscr{T}_{1}^{\prime}$ at every point is spanned by the $\frac{\partial}{\partial z^{\prime \prime}}$ directions : $\mathscr{T}_{1}^{\prime}=\left(\frac{\partial}{\partial z^{\prime \prime}}\right)$ for short. Extend $z^{\prime}, z^{\prime \prime}$ to $G$ and complete with coordinates $t=\left(t^{\prime}, t^{\prime \prime}\right)$ so that $t=0$ on $G^{\prime}$ and $\mathscr{T}_{2}=\left(\frac{\partial}{\partial t^{\prime}}\right)$ at every point of $G^{\prime}$. 
Then extend the coordinates $z^{\prime}, z^{\prime \prime}, t$ to $H$ and add one coordinate $z^{\prime \prime \prime}$ in such a way that $z^{\prime \prime \prime}=0$ on $G$ and $H_{f_{1}}=\frac{\partial}{\partial z^{\prime \prime \prime}}$ on $H$. Since $\Gamma$ is invariant under the symplectic flow generated by $H_{f_{1}}$ we have $t=0$ on $\Gamma$ and $\mathscr{T}_{1}=\left(\frac{\partial}{\partial z^{\prime \prime}}, \frac{\partial}{\partial z^{\prime \prime \prime}}\right)$ on $\Gamma$. Moreover if we redefine $\mathscr{T}_{2}$ outside $G^{\prime}$ on $\Gamma$ as $\left(\frac{\partial}{\partial t^{\prime}}\right)$ then we still have $T H \cap T \Gamma^{\perp}=\mathscr{T}_{1} \oplus \mathscr{T}_{2}$. Finally we extend $z, t$ to a full neighborhood of $\left(x_{0}, \xi_{0}\right)$ and add a coordinate $s$ such that $s=0$ on $H$ and $H_{f_{2}}=\left(\frac{\partial}{\partial s}\right)$. Since $g=\mathscr{O}\left((t, s)^{2{ }^{\prime \prime}}\right)$ and $T \Gamma^{\perp}=\left(\frac{\partial}{\partial z^{\prime \prime}}, \frac{\partial}{\partial z^{\prime \prime \prime}}, \frac{\partial}{\partial t^{\prime}}, \frac{\partial}{\partial s}\right)$ we have

$$
H_{g}=\mathscr{O}\left((t, s)^{z^{z-1}}\right)\left(\frac{\partial}{\partial z^{\prime \prime}}, \frac{\partial}{\partial z^{\prime \prime \prime}}, \frac{\partial}{\partial t^{\prime}}, \frac{\partial}{\partial s}\right)+\mathscr{O}\left((t, s)^{* z}\right)\left(\frac{\partial}{\partial z}, \frac{\partial}{\partial t}, \frac{\partial}{\partial s}\right) .
$$

Hence

$$
\begin{aligned}
H_{f_{1}}= & \frac{\partial}{\partial z^{\prime \prime \prime}}+\mathscr{O}\left(s(t, s)^{z-1}\right)\left(\frac{\partial}{\partial z^{\prime \prime}}, \frac{\partial}{\partial z^{\prime \prime \prime}}, \frac{\partial}{\partial t^{\prime}}, \frac{\partial}{\partial s}\right) \\
& +\mathscr{O}\left(s(t, s)^{\psi^{\prime \prime}}\right)\left(\frac{\partial}{\partial z}, \frac{\partial}{\partial t}, \frac{\partial}{\partial s}\right) .
\end{aligned}
$$

We next construct a function $\rho$ as before so that $\left.\rho\right|_{T \Gamma^{\perp}}=0$. We start with $\rho^{(0)}=\rho^{(0)}\left(z^{\prime}\right)$ on $\Gamma$. As an extension $\rho^{(1)}$ to $H$ we could take

$$
\rho^{(1)}(z, t)=\rho^{(0)}\left(z^{\prime}\right)+\vec{b}(z) t^{\prime \prime}+\mathscr{O}\left(t^{2}\right),
$$

but since $H_{f_{1}}(\rho)$ should vanish at least to the order $\mathscr{A}$ on $\Gamma$, we are obliged to take $\vec{b}=\vec{b}\left(z^{\prime}, z^{\prime \prime}\right)$. On the other hand our constructions seem to require a barrier in $\left\{z \in \Gamma ; \rho^{(0)}\left(z^{\prime}\right)=0\right\}$ which is not invariant under $z^{\prime \prime \prime}$-translation and $\vec{b}\left(z^{\prime}, z^{\prime \prime}\right)$ would have to vanish at this part of the battier. It thus seems difficult to make any interesting use of the $\vec{b} t^{\prime \prime}$ term so we drop it and put $\rho^{(1)}(z, t)=\rho^{(0)}\left(z^{\prime}\right)=\left|z^{\prime}\right|^{2}$.

Let $k(z)$ be a real valued analytic function on $\Gamma$ and put

$$
\boldsymbol{\rho}(\boldsymbol{z}, t, s)=\boldsymbol{\rho}^{(1)}(z, t)+k(z) \int_{0}^{s} \tilde{g}(z, t, \sigma) d \sigma=\left|z^{\prime}\right|^{2}+\mathscr{O}\left(s(t, s)^{* z}\right) .
$$

Here $\tilde{g}$ is an extension of $\left.g\right|_{\Sigma}$ which is of the order of maguitude $d_{\Gamma}^{*}$. Then

$$
H_{f_{2}}(\rho)=k(z) \tilde{g}(z, t, s),
$$

and using (4.29) we get easily

$$
H_{f_{1}}(\rho)=\mathcal{O}\left((t, s)^{*+1}\right) .
$$


Moreover by construction $H_{\rho} \in T \Gamma$ everywhere on $\Gamma$. Put

$$
r=\rho-\frac{H_{f_{1}}(\rho)}{\tilde{g}} f_{2}+\frac{H_{f_{2}}(\rho)}{\tilde{g}} f_{1}+R\left(f_{1}^{2}+f_{2}^{2}\right) .
$$

Then $r$ has the same regularity and holomorphic ectension properties as in the proof of the preceding theorem (and we do the same modification in the complex region as there). We have $H_{r} \in T \Sigma$ everywhere on $\Sigma, H_{r} \in T \Gamma$ everywhere on $\Gamma$ and on the set $z^{\prime}=0$ in $\Gamma$, which contains $\gamma_{0}$, we have $H_{r}=k H_{f_{1}}$. Defining $A, B, C$ as before we let $k$ vanish to the second order on $A$. Then $C$ is a barrier, because $r \sim\left|z^{\prime}\right|^{2}+f_{1}^{2}+f_{2}^{2}$ on this set, while $\nabla r=\mathscr{O}(1)\left(\left|z^{\prime}\right|+\left|f_{1}\right|+\left|f_{2}\right|\right)$ on $C$. (The only non-evident contribution to $\nabla r$ is $\frac{-H_{f_{1}}(\rho)}{\tilde{g}} \nabla f_{2}$, but $H_{f_{1}}(\rho)=\mathscr{O}\left((|t|+|s|)^{*+1}\left|z^{\prime}\right|\right)$ on $C$ so the estimate is easy). The proof is now completed as before.

We next show how to extend the Theorems 4.2-4. 4 to the case when $\operatorname{codim} \Sigma=d$ is even $>2$. As before we work near a point $\left(x_{0}, \xi_{0}\right) \in \Sigma$, where $\Sigma$ is non symplectic and we assume

$$
\operatorname{dim} T \Sigma \cap T \Sigma^{\perp} \leqslant 2 \text { everywhere on } \Sigma .
$$

If we represent $\Sigma$ by the $d$ real equations $f_{1}=\cdots=f_{d}=0$, and put $\mathscr{L}=$ $\left(\left\{f_{j}, f_{k}\right\}\right)_{1 \leqslant j, k \leqslant d}$ then $\pm \sqrt{\operatorname{det} \mathscr{L}}=g$ is a analytic function on $\Sigma$, well defined up to the sign, and more generally up to an elliptic factor, if we change the defining functions. (Indeed $\left.g=2^{d} d !\left(\Sigma\left\{f_{j}, f_{k}\right\} d x_{j} \wedge d x_{k}\right)^{d / 2} / d x_{1} \wedge \cdots \wedge d x_{d}\right)$. At the point $\left(x_{0}, \xi_{0}\right)$ we can make a linear recombination of $f_{1}, \cdots, f_{d}$ so that $T_{\left(x_{0}, \hat{\xi}_{0}\right)} \Sigma \cap T_{\left(x_{0}, \hat{\varepsilon}_{0}\right)} \Sigma^{\perp}$ is spanned by $H_{f_{1}}$ and $H_{f_{2}}$. Then $\Sigma_{s} ; f_{3}=\cdots=f_{d}=0$ is a symplectic manifold and after a symplectic change of coordinates we may assume that $\Sigma_{s}: x^{\prime \prime}=\xi^{\prime \prime}=0$. Changing the functions $f_{1}, \cdots, f_{d}$ once more we may assume that $f_{1}=f_{1}\left(x^{\prime}, \xi^{\prime}\right), f_{2}=f_{2}\left(x^{\prime}, \xi^{\prime}\right),\left(f_{3}, \cdots, f_{d}\right)=\left(x^{\prime \prime}, \xi^{\prime \prime}\right)$. The function $g$ is then just the Poisson bracket of $f_{1}$ and $f_{2}$. If we make the geometric assumptions of one of our 3 results for the manifold $\Sigma \subset \boldsymbol{R}_{\left(x^{\prime}, \xi^{\prime}\right)}^{2(n-d)+2}$ given by $f_{1}=f_{2}=0$, then we construct our weight function $r\left(x^{\prime}, \xi^{\prime}\right)$ as before and simply extend it to be constant in the $\left(x^{\prime \prime}, \xi^{\prime \prime}\right)$ variables. Then $r$ is of class $C^{1,1}$ in the real domain, and if $\Gamma \subset \Sigma$ is as before then we have a $C^{1,1}$ extension to a full complex neighborhood of $\left(x_{0}, \xi_{0}\right)$, holomorphic in a set of the form $\left|\operatorname{Im} \vec{g}\left(x^{\prime}, \xi^{\prime}\right)\right|<\varepsilon\left|\operatorname{Re} \vec{g}\left(x^{\prime}, \xi^{\prime}\right)\right|$ where $\vec{g}=\left(g_{1}, \cdots, g_{k}\right)$ are real defining functions of $\Gamma$. Since $H_{r}$ is tangent to $\Gamma, \hat{\Gamma}, \Sigma$ we can apply the remarks in the end of section 3. If $C \subset \boldsymbol{R}^{2(n-d)+2}$ is a barrier, then $C=\left\{(x, \xi) ;\left(x^{\prime}, \xi^{\prime}\right) \in C\right\}$ is also a barrier. The proofs of our theorems then go through without any further changes and we get:

THEOREM 4.2'. Let $\Sigma$ be of even codimension, assume (4.33) and define 
$g$ on $\Sigma$ as above. Let $\Gamma \subset \Sigma$ be an analytic hypersurface containing $\left(x_{0}, \xi_{0}\right)$ and assume (4.2) and (4.3). Let $\gamma_{0}$ be an integral curve segment of $T \Gamma \cap$ $T \Gamma^{\perp}$. If $u \in \mathscr{D}^{\prime}\left(\boldsymbol{R}^{n}\right)$ and $\gamma_{0} \cap W F_{a}(P u)=\phi$, then either $\gamma_{0} \subset W F_{a}(u)$ or $\gamma_{0} \cap$ $W F_{a}(u)=\phi$.

THEOREM 4. $3^{\prime}$. Let $\Sigma$ be of even codimension, assume (4.33) and define $g$ on $\Sigma$ as above. Let $\Gamma \subset \Sigma$ be an analytic submanifold containing $\left(x_{0}, \xi_{0}\right)$ and assume (4.13)-(4.15).

(a) If $\Gamma$ is symplectic, $u \in \mathscr{D}^{\prime}\left(\boldsymbol{R}^{n}\right), \quad\left(x_{0}, \xi_{0}\right) \notin W F_{a}(P u)$, then $\left(x_{0}, \xi_{0}\right) \notin$ $W F_{a}(u)$.

(b) If $\Gamma$ is not symplectic, let $L$ be a connected bicaracteristic leaf of $\Gamma$ containing $\left(x_{0}, \xi_{0}\right) . \quad$ If $u \in \mathscr{D}^{\prime}\left(\boldsymbol{R}^{n}\right), L \cap W F_{a}(P u)=\phi$, then either $L \subset W F_{a}(u)$ or $L \cap W F_{a}(u)=\phi$.

THEOREM 4. $4^{\prime}$. Let $\Sigma$ be of even codimension, assume (4.33) and define $g$ on $\Sigma$ as above. Let $\Gamma \subset \Sigma$ be an analytic submanifold containing $\left(x_{0}, \xi_{0}\right)$ and assume (4.13), (4.15), (4.27). Let $\gamma_{0}$ be an integral curve segment of $T \Gamma \cap T \Sigma^{\perp}$ containing $\left(x_{0}, \xi_{0}\right)$. Then either $\gamma_{0} \subset W F_{a}(u)$ or $\gamma_{0} \cap W F_{a}(u)=\phi$.

As our last application, we shall consider an operator which is not $C^{\infty}$ hypoelliptic. Let $Q(x, D)$ be a differential operator with analytic coefficients, defined near $x_{0} \in \boldsymbol{R}^{n}$. Let $q$ be the principal symbol and assume that in a neighborhood of $\left(x_{0}, \xi_{0}\right) \in T^{*} \boldsymbol{R}^{n} \backslash 0$ :

(4.34) $q$ is real valued and vanishes to the second order on a symplectic submanifold $\Sigma \ni\left(x_{0}, \xi_{0}\right)$ which is of codimension $2 d$.

For $\rho \in \Sigma$ let $F_{\rho}$ be the fundamental matrix of $q$, defined by $q^{\prime \prime}(t, s)=\sigma\left(t, F_{\rho} s\right)$, $\forall t, s \in T_{\rho}\left(\boldsymbol{R}^{2 n}\right)$. Here the Hessian $q^{\prime \prime}$ and the symplectic form $\sigma$ are considered as bilinear forms. We assume

For all $\rho \in \Sigma, F_{\rho}$ is of rank $2 d$ and has no purely imaginary nonvanishing eigenvalues.

For $\rho \in \Sigma$, let $\Lambda_{+}(\rho), \Lambda_{-}(\rho) \subset T_{\rho}\left(\boldsymbol{R}^{2 n}\right)$ be the $d$-dimensional isotropic subspaces whose complecifications are the sum of all complex (generalized) eigenspaces corresponding to eigenvalues with positive respectively negative, real parts. Then $T_{\rho}(\Sigma)^{\perp}=\Lambda_{+}(\rho) \oplus \Lambda_{-}(\rho)$.

We then know (see appendix) that in a neighborhood of $\left(x_{0}, \xi_{0}\right)$ there are $H_{q}$-incariant, involutive manifolds $\mathscr{T}_{+}, \mathscr{T}_{-}$of codimension $d$, such that $\Sigma \subset \mathscr{T}_{ \pm}$and $T_{\rho}\left(\mathscr{T}_{ \pm}\right)=T_{\rho}(\Sigma) \oplus \Lambda_{ \pm}(\rho)$ at every point $\rho \in \Sigma$. Within $\mathscr{T}_{+}\left(\mathscr{T}_{-}\right)$, $\Sigma$ is a repulsive (attractive) submanifold for $H_{q}$, and $\left.q\right|_{J_{ \pm}}=0$. We can find symplectic coordinates $\left(x^{\prime}, x^{\prime \prime}, \xi^{\prime}, \xi^{\prime \prime}\right)$ centered at $\left(x_{0}, \xi_{0}\right)$ such that $\Sigma: x^{\prime \prime}=\xi^{\prime \prime}$ $=0, \mathscr{T}_{+}: \xi^{\prime \prime}=0, \mathscr{T}_{-}: x^{\prime \prime}=0$; The principal symbol $q$ becomes 


$$
q(x, \xi)=A(x, \xi) x^{\prime \prime} \cdot \xi^{\prime \prime}
$$

where $A$ is a real $d \times d$-matrix. At a point $\rho \in \Sigma$ the fundamental matrix restricted to $T_{\rho} \Sigma^{\perp} \simeq \boldsymbol{R}_{\left(x^{\prime \prime}, \xi^{\prime \prime}\right)}^{2 d}$ becomes $\left(\begin{array}{cc}A & 0 \\ 0 & -{ }^{t} A\end{array}\right)$ and the eigenvalues $\lambda_{1}, \cdots, \lambda_{d}$ of $A$ are precisely those of $F$ which have real parts $>0$. Let $\lambda_{ \pm} \subset \mathscr{T}_{ \pm}$be the bicharacteristic leaves through $\left(x_{0}, \xi_{0}\right)=(0,0)$ so that $\lambda_{+}: x^{\prime}=0, \xi=0, \lambda_{-}$: $\xi^{\prime}=0, x=0$. We write

$$
H_{q}=A(x, \xi) x^{\prime \prime} \cdot \frac{\partial}{\partial x^{\prime \prime}}-{ }^{t} A(x, \xi) \xi^{\prime \prime} \cdot \frac{\partial}{\partial \xi^{\prime \prime}}+\mathcal{O}\left(\left(x^{\prime \prime}\right)\left(\xi^{\prime \prime}\right)\right) .
$$

(Here $\mathcal{O}\left(\left(x^{\prime \prime}\right)\left(\xi^{\prime \prime}\right)\right)$ indicates a term with coefficients in the product of the two ideals generated respectively by $x_{d+1}, \cdots, x_{n}$ and $\xi_{d+1}, \cdots, \xi_{n}$.) Let $A_{0}=$ $A(0,0)$ and put

$$
B=\int_{0}^{\infty} e^{-s^{t} A_{0}} e^{-s A_{0}} d s
$$

which is a positive symetric matrix with the property that

$$
{ }^{t} A_{0} B+B A_{0}=I .
$$

If $\left\|x^{\prime \prime}\right\|^{2}=\left\langle B x^{\prime \prime}, x^{\prime \prime}\right\rangle$ is the corresponding norm, then

$$
A_{0} x^{\prime \prime} \cdot \frac{\partial}{\partial x^{\prime \prime}}\left(\left\|x^{\prime \prime}\right\|^{2}\right)=\left\|x^{\prime \prime}\right\|^{2}>0,
$$

where $\left\|x^{\prime \prime}\right\|$ is the standard norm. Similary we can find a symetric matrix $C>0$ so that

$$
{ }^{t} A_{0} \xi^{\prime \prime} \cdot \frac{\partial}{\partial \xi^{\prime \prime}}\left(\left\|\xi^{\prime \prime}\right\|_{*}^{2}\right)=\left\|\xi^{\prime \prime}\right\|^{2}>0,
$$

if $\left\|\xi^{\prime \prime}\right\|_{*}^{2}=\left\langle C \xi^{\prime \prime}, \xi^{\prime \prime}\right\rangle$.

Let $[0, T] \ni t \rightarrow(x(t), \xi(t))$ be an integral curve of $H_{q}$, with $|(x(0), \xi(0))| \leqslant \varepsilon$, $\left\|x^{\prime \prime}(0)\right\|>\frac{1}{2}\left\|\xi^{\prime \prime}(0)\right\| *,\left\|x^{\prime \prime}(t)\right\| \leqslant \delta_{0}$, where $\delta_{0}>0$ is small but independent of $\varepsilon$. Then with a constant $C_{0}>0$ independent of $\varepsilon$ we get

$$
\begin{aligned}
& \left\|\xi^{\prime \prime}(t)\right\| \leqslant e^{-t / C_{0}}\left\|\xi^{\prime \prime}(0)\right\| \leqslant C_{0} \varepsilon e^{-t / C_{0}}, \\
& \left\|x^{\prime \prime}(t)\right\| \geqslant e^{t / C_{0}}\left\|x^{\prime \prime}(0)\right\|, \quad\left|\frac{d}{d t}\left(x^{\prime}(t), \xi^{\prime}(t)\right)\right| \leqslant C_{0} \varepsilon e^{-t / C_{0}} .
\end{aligned}
$$

From this we see that we can increase $T$ so that $\left\|x^{\prime \prime}(T)\right\|=\delta_{0}$ and $\left|\left(x^{\prime}(t), \xi^{\prime}(t)\right)\right|+\left|\xi^{\prime \prime}(t)\right| \leqslant$ const. $\varepsilon, 0 \leqslant t \leqslant T$. Hence the distance from $(x(t), \xi(t))$ to $\lambda_{+}$is at most; (const.) s. Naturally we have an analogous result if $\left\|\xi^{\prime \prime}(0)\right\|_{*}>\frac{1}{2}$ 
$\left\|x^{\prime \prime}(0)\right\| \|$ and we study the integral curve for negative times, provided that we replace $\lambda_{+}$by $\lambda_{-}$. We shall state and prove a theorem under the assumption

$$
\left.\left(x_{0}, \xi_{0}\right) \notin W F_{a}(Q u), \quad W F_{a} u\right) \cup\left(\lambda_{-} \backslash\left\{\left(x_{0}, \xi_{0}\right)\right\}\right)=\phi .
$$

Then the above discussion and the fact that analytic regularity propagates along the integral curves of $H_{q}$ show that there exists $\varepsilon>0$ such that in our special coordinates :

$$
\left\{(x, \xi) \in \boldsymbol{R}^{2 n} ;|(x, \xi)| \leqslant \varepsilon,\left\|\xi^{\prime \prime}\right\|_{*}>\frac{1}{2} \| x^{\prime \prime}\right\} \cap W F_{a}(u)=\phi .
$$

Let $S_{Q}$ be the subprincipal symbol of $Q$, invariantly defined on $\Sigma$ and define $\lambda_{1}, \cdots, \lambda_{d}$ as above. We assume

$$
S_{Q}+\frac{1}{i} \sum_{1}^{d}\left(\alpha_{j}+\frac{1}{2}\right) \lambda_{j} \neq 0
$$

everywhere on $\Sigma$, for all $\alpha_{j} \in\{0,1,2, \cdots\}$. The folowing result is due to Ôaku [13] in the case when $\operatorname{codim} \Sigma=2$.

THEOREM 4.5. Let $Q$ have the properties above, in particular (4.34), (4. 35), (4.40), and let $\lambda_{+} ; \lambda_{-}, \Sigma,\left(x_{0}, \xi_{0}\right)$ be as above. If $u \in \mathscr{D}^{\prime}\left(\boldsymbol{R}^{n}\right)$ and (4.38) holds, then $\left(x_{0}, \xi_{0}\right) \notin W F_{a}(u)$.

Proof. In the special coordinates above, put

$$
r(x, \xi)=\left\|x^{\prime \prime}\right\|^{2}-\left\|\xi^{\prime \prime}\right\|_{*}^{2}+\left|x^{\prime}\right|^{2}+\left|\xi^{\prime}\right|^{2} .
$$

Then $H_{r}$ is tangent to $\Sigma,(4.37)$ and the discussion there after show that

$$
H_{q}(r)=\left\|x^{\prime \prime}\right\|^{2}+\left\|\xi^{\prime \prime}\right\|^{2}+\mathcal{O}\left(\left(x^{\prime \prime}, \xi^{\prime \prime}\right)^{2}(x, \xi)\right) .
$$

Hence on the real domain :

$$
\begin{aligned}
& q \circ \exp \left(i t H_{r}\right)=q(x, \xi)-i t\left(\left\|x^{\prime \prime}\right\|^{2}+\left\|\xi^{\prime \prime}\right\|^{2}+\mathscr{O}\left(\left(x^{\prime \prime}, \xi^{\prime \prime}\right)^{2}(x, \xi)\right)\right) \\
& \quad+\mathscr{O}\left(t^{2}\left(x^{\prime \prime}, \xi^{\prime \prime}\right)^{2}\right),
\end{aligned}
$$

so for $t>0$ small enough,

$$
\left|\operatorname{Re} q \circ \exp \left(i t H_{r}\right)\right| \leqslant \frac{C}{t}\left(-\operatorname{Im} q \circ \exp \left(-i t H_{r}\right)\right) .
$$

We noticed above that (4.39) follows from (4.38) so we see that the set $r \leqslant 0$ is disjoint from $W F_{a}(u)$ in a pointed real neighborhood of $\left(x_{0}, \xi_{0}\right)=(0,0)$.

We now perform our usual FBI-transform and use the same notation for the transformed objects. As before let $\varphi_{t}(x)=\varphi(t, x)$ be the solution to the 
problem (3.7). Then if $\varepsilon>0$ is small enough, we-have $u \sim 0$ in $H_{\varphi_{t}}^{\text {loc }}$ in a neighborhood of $\left|x-x_{0}\right|=\varepsilon$, when $t>0$ is small enough. Indeed at those points, either $r\left(x, \frac{2}{i} \frac{\partial \varphi_{0}}{\partial x}\right)>0$ so $\varphi_{\mathrm{t}}>\varphi_{0}$ and there is nothing to prove, or $u \sim 0$ in $H_{\varphi_{0}}$. Since $\varphi_{t}\left(x_{0}\right)=\varphi_{0}\left(x_{0}\right)$ it suffices to show that the conclusion of Theorem 2. 1 is valid with $\varphi=\varphi_{t}$, in order to conclude that $u \sim 0$ in $H_{\varphi_{0}}$ at $x_{0}$ (and hence that $\left(x_{0}, \xi_{0}\right) \notin W F_{a}(u)$ in the untransformed notation). However, the proof of Theorem 2. 1 goes through without changes if we consider $\varphi_{t}$ as the unperturbed weight (and take $t>0$ small). (4.43) shows that $|q|_{\Lambda_{\varphi_{t}}}$ behaves like the square of the distance to $\Lambda_{\varphi_{t}} \cap \Sigma^{C}$ so the estimates in the elliptic region are unchanged. (4.44) shows that $\left.q\right|_{\Lambda_{\varphi_{t}}}$ takes its values in an angle $\left\{z \in C ;\left|\arg z+\frac{\pi}{2}\right| \leqslant \alpha\right\}, \alpha<\frac{\pi}{2}$, and (4.40) then gives an explicit and wellknown condition which allows us to obtain (2.27), using only the earlier arguments. The proof is complete.

We end this section by giving some examples to the Theorems 4.2-4.4. Let $U$ be an open set in $\boldsymbol{R}^{n}$ or possibly an $n$-dimensional manifold and $\Sigma=\{(x, \lambda \rho(x)) ; \lambda \in \boldsymbol{R}, x \in U\}$ where $\rho$ is a non-vanishing 1 -form on $U$. To fix : the ideas, we may assume that $P$ is a second order differential operator with principal symbol $\geqslant 0$ which locally behaves like the square of the distance to $\Sigma$. We also assume through out that $P$ is hypoelliptic with loss of 1 derivative. If $\omega=\sum_{1}^{n} \xi_{j} d x_{j}$ is the fundamental 1 -form and we consider $\rho(x)$ as 1 -form on $\Sigma$ in the natural way, then $\left.\omega\right|_{\Sigma}=\lambda \rho$ and hence $\left.\sigma\right|_{\Sigma}=\lambda d \rho+$ $d \lambda \wedge \rho$, if $\sigma=d \omega$ is the symplectic form. Let $\left(t_{x}, t_{\lambda}\right),\left(s_{x}, s_{\lambda}\right)$ be tangent vectors to $\Sigma$ at a given point. Then

$$
\begin{aligned}
\left\langle\left.\sigma\right|_{\Sigma},\right. & \left.\left(t_{x}, t_{\lambda}\right) \wedge\left(s_{x}, s_{\lambda}\right)\right\rangle= \\
& =\lambda\left\langle d \rho, t_{x} \wedge s_{x}\right\rangle+t_{\lambda}\left\langle\rho, s_{x}\right\rangle-s_{\lambda}\left\langle\rho, t_{x}\right\rangle .
\end{aligned}
$$

Let $H \subset U$ be a submanifold and let $\Gamma=\{(x, \lambda \rho(x)) ; x \in H\}$ be the corresponding submanifold of $\Sigma$. We shall only consider 2 pure cases :

(a) $\left.\rho\right|_{H}=0$. Then (4.45) shows that $\Gamma$ is isotropic.

(b) $\left.\rho\right|_{H} \neq 0$ everywhere. Then $\left(0, t_{2}\right)$ cannot be in the kernel of $\left.\sigma\right|_{\Sigma}$ unless $t_{2}=0$. On the other hand, for $\left(t_{x}, t_{2}\right)$ to be in the kernel of $\left.\sigma\right|_{r}$ at a point $\left(x_{0}, \lambda_{0}\right)$, the necessary and sufficient condition on $t_{x}$ is that $t_{x} \in \operatorname{Ker} \rho \cap$ $T H$ and that $t_{x}$ is also in the kernel of $d_{\rho}$ restricted to $(\operatorname{Ker} \rho \cap T H) \times(\operatorname{Ker} \rho \cap$ $T H)$. For such vectors $t_{x}$ there is a unique $t_{2}$ such that $\left.\left(t_{x}, t_{2}\right) \in \operatorname{Ker} \sigma\right|_{r}$.

Let $\Omega \subset C^{n}$ be given by $f(z)<0$, where $f$ is real and analytic with $d f \neq 0$ on $\partial \Omega$. We are interested in the case when $P$ has the same characteristic variety as the Kohn-Laplacian $\square_{b}$. This means that we can take $U=\partial \Omega$ 
and $\rho=\left.\frac{1}{i} \partial f\right|_{\partial \Omega}$ so $d \rho=\left.\frac{1}{i} \bar{\partial} \partial f\right|_{\partial \Omega}$ is the Levi form. Recall in this situation that the complexification of $\operatorname{Ker} \rho$ is $T^{1,0}(\partial \Omega) \oplus T^{0,1}(\partial \Omega)$, where $T^{1,0}(\partial \Omega)\left(T^{0,1}(\partial \Omega)\right)$ is the bundle of holomorphic (anti-holomorphic) tangent vectors. Let $Z_{1}, \cdots$, $Z_{n-1}$ be linearly independent local sections of $T^{1,0}(\partial \Omega)$. Then we recall that

$$
\left\langle\frac{1}{i} \ddot{\partial} \partial f, \bar{Z}_{j} \wedge Z_{k}\right\rangle=\left\langle\rho,\left[\bar{Z}_{j}, Z_{k}\right]\right\rangle \stackrel{\text { def }}{=} a_{j k}
$$

and that

$$
\left.\frac{1}{i} \bar{\partial} \partial f\right|_{\operatorname{Ker}_{\rho} \times \operatorname{Ker} \rho}=\sum a_{j k} \bar{u}_{j} \wedge u_{k}
$$

where $u_{1}, \cdots, u_{n-1}$ is the dual system of $(1,0)$-forms on $\operatorname{Ker} \rho$. If $g_{j}$ is the principal symbol of $Z_{j}$ then the matrix $a_{j k}$ is up to an elliptic factor the same as

$$
G=\left.\left(\frac{1}{i}\left\{\bar{g}_{j}, g_{k}\right\}\right)\right|_{\Sigma} \cdot
$$

The function $g$, discussed earlier in this section, is then the determinant of $G$. Indeed $\Sigma$ is given by $f_{1}=\cdots=f_{2 d}=0$ where $f_{j}=g_{j}, 1 \leqslant j \leqslant d, f_{j}=\bar{g}_{j-d}$, $d+1 \leqslant j \leqslant 2 d$ and

$$
\left(\frac{1}{i}\left\{f_{j}, f_{k}\right\}\right)=\left(\begin{array}{cc}
0 & -{ }^{t} G \\
G & 0
\end{array}\right) .
$$

(The functions $f_{j}$ are not real valued, but their differentials are linearly independent over $\boldsymbol{C}$, which is all that is needed here).

$1^{\circ}$. Let $n=2$ and let $H \subset \partial \Omega$ be a curve on which the function $g$ vanishes. If $\left.\rho\right|_{H} \neq 0$ everywhere, then $\Gamma$ is symplectic by (4.45) and Theorem 4.3 (a) gives analytic regularity provided that $g \sim d_{H}^{2 k} k \in\{1,2, \cdots\}$. An example of this situation is when $\Omega:\left|z_{1}\right|^{2}+\left|z_{2}\right|^{4} \leqslant 1$ and $H \subset \partial \Omega$ is given by $z_{2}=0$. Then $T^{1,0}(\partial \Omega)=\left(\frac{\partial}{\partial z_{2}}\right)$ at the points of $H$ and $g \sim d_{H}^{2}$.

$2^{\circ}$. Let $n=2$ and let $H \subset \partial \Omega$ be a curve on which $g$ vanishes. If $\left.o\right|_{H}=0$ everywhere, then $\Gamma$ is isotropic by (4.45) and we may choose $Z=X+i Y$ such that $X$ is tangent to $H$ (while $Y$ is non-tangent). With $f_{1}, f_{2}=$ principal symbol of $X, Y$ we have $\Pi_{x}\left(H_{f_{1}}\right)$ tangent to $H, H_{f_{1}}$ tangent to $\Sigma$, so $H_{f_{1}}$ is tangent to $\Gamma$, while $H_{f_{2}}$ is not tangent to $\Gamma$. If the function $g$ has the right degeneration on $H$, we may apply Theorem 4.4 to deduce propagation of analytic regularity along $H$. An example of this situation is $\Omega: \operatorname{Re}\left(z_{1}^{2}+z_{2}^{2}\right)$ $+\left(y_{1}^{2}+y_{2}^{2}\right)^{2} \leqslant 1$, and $H \subset \partial \Omega$ given by $y_{1}=y_{2}=0$. The complete Levi-matrix of the function $f$, then vanishes for $y=0$ and satisfies $\mathscr{L}_{f} \geqslant 2 y^{2} \mathscr{L}_{y^{2}}$. (We define 
$\left.\mathscr{L}_{f}=\left(\frac{\partial^{2} f}{\partial \bar{z}_{j} \partial z_{j}}\right)\right)$. It is therefore clear that $g \sim y^{2}$. On $H$ we have $T^{1,0}(\partial \Omega)=$ $\left(x_{2} \frac{\partial}{\partial z_{1}}-x_{1} \frac{\partial}{\partial z_{2}}\right)$ and clearly $\left.\rho\right|_{H}=0$.

$3^{\circ}$. Let $n=2$ and let $H \subset \partial \Omega$ be a hypersurface on which $g$ vanishes. The case when $H$ is a complex curve has already been treated by GrigisSchapira-Sjöstrand [3], Métivier [12], and we assume instead that the dimension of $T H \cap \operatorname{Ker} \rho$ is equal to 1 everywhere on $H$. With the same notation as in $2^{\circ}$ we may assume that this space is given by $\Pi_{x}\left(H_{f_{1}}\right)$, so that $h_{f_{1}}$ is tangent to $\Gamma$ while $H_{f_{2}}$ is transverse to $\Gamma$. From (4.45) and the discussion there after it is clear that $\left.\operatorname{Ker} \sigma\right|_{r}$ is of dimension 1 and generated' by $H_{f_{1}}$. Depending on wether $g$ degenerates to odd or even order on $\Gamma$ we may either apply Theorem 4.2 or Theorem 4.4, and conclude that analytic regularity propagates in the $\Pi_{x}\left(H_{f_{1}}\right)$-direction in $H$. An example of this is when $\Omega$ is given by $y_{2}>y_{1}^{k}, k \geqslant 3$ and $H \subset \partial \Omega$ is given by $y_{1}=0$. At an arbitrary point of $\partial \Omega$ we have $T^{1,0}(\partial \Omega)=\left(\frac{\partial}{\partial z_{1}}+k y_{1}^{k-1} \frac{\partial}{\partial z_{2}}\right)$ and $g$ vanishes on $H$ to the order $k-2$ precisely. Moreover $T H \cap \operatorname{Ker} \rho=\left(\frac{\partial}{\partial x_{1}}\right)$.

$4^{\circ}$. Let $n=3$ and let $H \subset \partial \Omega$ be a curve with $\left.\rho\right|_{H}=0$, on which $\left.\frac{1}{i} \bar{\partial} \partial f\right|_{\text {Ker } \rho \times \operatorname{Ker} \rho}$ is of rank 2. We assume that the tangent of $H$ is not in the kernel of this restricted 2 -form. Then $\Gamma$ is an isotropic submanifold of $\Sigma$ with $T \Gamma \cap T \Sigma^{\perp}=0$, while $T \Sigma \cap T \Sigma^{\perp}$ is of dimension 2 along $\Gamma$. If the function $g$ is locally of the same order of maguitude as $d_{H}^{2 k}, k \in\{1,2, \cdots\}$, then we can apply Theorem 4.3 (b) and conclude that analytic regularity propagates along $H$. As an example of this situation we may define $\Omega$ in $C^{3}$ by $\left|z_{1}\right|^{2}+\left|z_{2}\right|^{2}+\left(y_{1}^{2}+y_{2}^{2}+\left|z_{3}\right|^{2}\right)^{2} \leqslant 1$ and let $H \subset \partial \Omega$ be the circel given by $y_{1}=y_{2}=0, z_{3}=0$. Then on $H$ we have $T^{1,0}(\partial \Omega)=\left(\frac{\partial}{\partial z^{3}}, x_{2} \frac{\partial}{\partial z_{1}}-x_{1} \frac{\partial}{\partial z_{2}}\right)$, $\left.\rho\right|_{H}=0$, while $\frac{1}{i} \bar{\partial} \partial f=\frac{1}{i}\left(\overline{d z_{1}} \wedge d z_{1}+\overline{d z_{2}} \wedge d z_{2}\right)$ is of rank 2 on $\operatorname{Ker} \rho \times \operatorname{Ker} \rho$, $\operatorname{Ker} \rho=\left(\frac{\partial}{\partial x_{3}}, \frac{\partial}{\partial y_{3}}, x_{2} \frac{\partial}{\partial x_{1}}-x_{1} \frac{\partial}{\partial x_{2}}, x_{2} \frac{\partial}{\partial y_{1}}-x_{1} \frac{\partial}{\partial y_{2}}\right)$. The complete Levimatrix of $f$ is $\geqslant \mathscr{L}_{\left|z_{1}\right|^{2}+\left|z_{z^{2}}\right|^{2}}+2\left(y_{1}^{2}+y_{2}^{2}+\left|z_{3}\right|^{2}\right) \mathscr{L}_{\left|z_{3}\right|^{2}}$ so the function $g$ is of the same order of maguitude as $y_{1}^{2}+y_{2}^{2}+\left|z_{3}\right|^{2}$. 


\section{APPENDIX}

In the situation described before Theorem 4.5, we are going to establish the local existence of involutive analytic manifolds $\mathscr{T}_{ \pm}$of codimension $d$, such that $H_{q}$ is tangent to $\mathscr{T}_{ \pm}, \Sigma \subset \mathscr{T}_{ \pm}$and $T_{\rho}\left(\mathscr{T}_{ \pm}\right)=T_{\rho}(\Sigma)+\Lambda_{ \pm}(\rho)$ for every $\rho \in \Sigma$. At least in the $C^{\infty}$-case this can be regarded as a consequence of known results about stable manifolds (see Abraham-Marsden [1]). Nevertheless it might be useful to give a rather short proof in the analytic case, somewhat in the spirit of B. Lascar-Sjöstrand [9].

We may first choose real, analytic coordinates $\left(x, y^{\prime \prime}\right)$, with $x=\left(x^{\prime}, x^{\prime \prime}\right)$ centered at $\rho_{0} \in \Sigma$, such that $\Sigma$ is given by $x^{\prime \prime}=y^{\prime \prime}=0$ and such that $v=H_{q}$ is given by

$$
v=A x^{\prime \prime} \cdot \frac{\partial}{\partial x^{\prime \prime}}-A_{*} y^{\prime \prime} \cdot \frac{\partial}{\partial y^{\prime \prime}}+\mathcal{O}\left(\left(x, y^{\prime \prime}\right)\left(x^{\prime \prime}, y^{\prime \prime}\right)\right)\left(\frac{\partial}{\partial x}, \frac{\partial}{\partial y^{\prime \prime}}\right) .
$$

Here $S p(A), S p\left(A_{*}\right) \subset\{\operatorname{Re} \lambda>0\}$. As we saw in section 4 , there are norms $\left\|x^{\prime}\right\|,\left\|{ }^{\prime \prime} y^{\prime \prime}\right\| *$ such that

$$
A x^{\prime \prime} \cdot \frac{\partial}{\partial x^{\prime \prime}}\left\|x^{\prime \prime}\right\| \geqslant C\left\|x^{\prime \prime}\right\|, A_{*} y^{\prime \prime} \cdot \frac{\partial}{\partial y^{\prime \prime}}\left\|y^{\prime \prime}\right\|_{*} \geqslant C ! \mid y^{\prime \prime} \|_{*},
$$

where $C>0$. These inequalities are valid also in the complex domain for the natural "hermitian" extensions of the norms provided that we consider the associated real vector fields $A x^{\prime \prime} \cdot \frac{\partial}{\partial x^{\prime \prime}}, A_{*} y^{\prime \prime} \cdot \frac{\partial}{\partial y^{\prime \prime}}$ instead. We now restrict the attention to a complex region of the form

$$
\Omega=\left\{\left(x, y^{\prime \prime}\right) ;\left\|y^{\prime \prime}\right\|_{*}<\left\|x^{\prime \prime}\right\|<f\left(\left|x^{\prime}\right|^{2}\right)\right\},
$$

where $0 \leqslant f \in C_{0}^{\infty}\left(\overline{\boldsymbol{R}}_{+}\right)$is $>0$ at 0 , has a sufficiently small support and a Sufficiently small derivative everywhere. From (A.1) it follows that

$$
\begin{aligned}
& \hat{v}\left(\left\|x^{\prime \prime}\right\|\right) \geqslant \frac{C}{2}\left\|x^{\prime \prime}\right\| \quad \text { in } \Omega, \\
& \hat{v}\left(\left\|y^{\prime \prime}\right\|_{*}\right) \leqslant-\frac{C}{2}\left\|y^{\prime \prime}\right\|_{*} \text { on } \partial \Omega \cap\left\{\left\|y^{\prime \prime}\right\|\left\|_{*}=\right\| x^{\prime \prime} \|\right\} .
\end{aligned}
$$

Since we also have $\hat{v}\left(x^{\prime}\right)=\mathscr{O}\left(\left(x, y^{\prime \prime}\right)\left(x^{\prime \prime}, y^{\prime \prime}\right)\right)$ and the gradient of $f$ is small, we see that for every $\rho \in \Omega$, there is a $T(\rho)>0$ such that $\Phi_{t}(\rho) \stackrel{\text { def }}{=} \exp t \hat{v}(\rho) \in \Omega$ for $0 \leqslant t<T(\rho), \Phi_{T(\rho)}(\rho) \in \partial \Omega \cap\left\{\left\|x^{\prime \prime}\right\| \|=f\left(\left.\left|x^{\prime}\right|\right|^{2}\right)\right\}$, while $\Phi_{t}(\rho) \notin \Omega$ for $T(\rho) \leqslant t \leqslant$ $T(\rho)+\frac{1}{C}$. Here $C>0$ is independent of $\rho$. 
The evolution of a tangent vector $\left(\delta_{x}(t), \delta_{y^{\prime \prime}}(t)\right)$ along an integral curve of $\hat{v}$ is given by

$$
\left\{\begin{array}{l}
\dot{\delta}_{x^{\prime \prime}}=A \delta_{x^{\prime \prime}}+\mathscr{O}\left(\left(x, y^{\prime \prime}\right)\right)\left(\delta_{x}, \delta_{y^{\prime \prime}}\right) \\
\dot{\delta}_{y^{\prime \prime}}=-A_{*} \delta_{y^{\prime \prime}}+\mathscr{O}\left(\left(x, y^{\prime \prime}\right)\right)\left(\delta_{x}, \delta_{y^{\prime \prime}}\right) \\
\dot{\delta}_{x^{\prime}}=0+\mathcal{O}\left(\left(x, y^{\prime \prime}\right)\right)\left(\delta_{x}, \delta_{y^{\prime \prime}}\right)
\end{array}\right.
$$

If $\varepsilon_{0}>0$, and $\Omega$ is sufficiently small, then the region, given by

$$
\left\|\delta_{y^{\prime}}\right\|_{*}^{2} \leqslant \varepsilon_{0}^{2}\left(\left\|\delta_{x^{\prime}},\right\| \|^{2}+\left|\delta_{x^{\prime}}\right|^{2}\right) \text {. }
$$

is stable under the evolution along an integral curve in $\Omega$, when the time increases.

Let $\mathscr{T}_{0}: y^{\prime \prime}=0, x^{\prime \prime} \in \Pi(\Omega)$, where $\Pi(\Omega)$ is given by $\left\|x^{\prime \prime}\right\|<f\left(\left|x^{\prime}\right|^{2}\right)$. Let $\mathscr{T}_{t}=\Phi_{t}\left(\mathscr{T}_{0}\right) \cap \Omega, t \geqslant 0$. Then using the above remarks, we see that $\mathscr{T}_{t}$ is given by $y^{\prime \prime}=g_{t}(x)$, where $g_{t}$ is holomorphic in $\Pi(\Omega)$, (A. 5) holds for every tangent vector to $\mathscr{T}_{t}, g_{t}\left(x^{\prime}, 0\right)=0$ and $\left\|g_{t}(x)\right\|\left\|_{*} \leqslant \frac{1}{2}\right\| x^{\prime \prime} \|$. By compactness, there is a sequence $t_{j} \rightarrow+\infty$ such that $g_{t_{j}} \rightarrow g$ in the space of holomorphic functions on $\Pi(\Omega)$. We put $\mathscr{T}_{+}=\{(x, g(x)) ; x \in \Pi(\Omega)\}$.

Let $\rho_{t}$ be an integral curve of $\hat{v}$, not in $\mathscr{T}_{t}$. Fix $t=t_{0}$ and let $\gamma_{t_{0}}$ be the shortest segment joining $\varphi_{t_{0}}$ to $\mathscr{T}_{t_{0}}$. (We use the metric $\left|\delta_{x^{\prime}}\right|^{2}+\left\|\delta_{x^{\prime}}\right\| \|^{2}+$ \|\|$\delta_{y^{\prime}}, \|_{*}^{2}$ ). Since (A 5) holds for every tangent vector of $\mathscr{T}_{t_{0}}$ and $\gamma_{t_{0}}$ is orthogonal to $T\left(\mathscr{T}_{t_{0}}\right)$ at the point of intersection with $\mathscr{T}_{t_{0}}$, we see that the directional vector $\nu$ of $\gamma_{t_{0}}$ satisfies

$$
\left\|\nu_{y^{\prime}},\right\| \|_{*}^{2} \geqslant \varepsilon_{0}^{-2}\left(\left|\nu_{x^{\prime}}\right|^{2}+\left\|\nu_{x^{\prime}},\right\| \|^{2}\right) \text {. }
$$

In the region (A. 6) the evolution (A. 4) is contractive, hence $\frac{d}{d t}\left|\gamma_{t}\right| \leqslant-C\left|\gamma_{t}\right|$ at $t=t_{0}$, if $\left|\gamma_{t}\right|$ denotes the length. We conclude that

$$
\frac{d}{d t} d\left(\rho_{t}, \mathscr{T}_{t}\right) \leqslant-C d\left(\rho_{t}, \mathscr{T}_{t}\right)
$$

for all $t$. so the integral curves of $\hat{v}$ approach $\mathscr{T}_{t}$ exponentially fast at $t$ increases. It follows that $\left|g_{t+s}-g_{t}\right| \leqslant C e^{-t / C}$ for $s, t \geqslant 0$ and hence that $g_{t} \rightarrow g$ exponentially fast Now it is clear that $\mathscr{T}_{+}$is invariant under the flow and that (A 7) holds with $\mathscr{T}_{t}$ replaced by $\mathscr{T}_{+}$. We can then caracterize $\mathscr{T}_{+}$ as the set of $\rho \in \Omega$ such that $\Phi_{-t}(\rho) \in \Omega$ for all $t \geqslant 0$. (moreover $\Phi_{-t}(\rho) \rightarrow \Sigma^{c}$, $t \rightarrow+\infty$ when $\left.\rho \in \mathscr{T}_{+}\right)$. Moreover $q=0$ on $\mathscr{T}_{+}$. If $u_{1}, u_{2}$ are holomorphic functions vanishing on $\mathscr{T}_{+}$and $\rho \in \mathscr{T}_{+}$, then $\left\{u_{1}, u_{2}\right\}(\rho)=\left\{u_{1} \circ \Phi_{t}, u_{2} \circ \Phi_{t}\right\}\left(\Phi_{-t}(\rho)\right)$. 
Since integral curves of $\hat{v}$ approach $\mathscr{T}_{+}$exponentially fast we see that $d u_{j} \circ \Phi_{t}\left(\Phi_{-t}(\rho)\right)$ tends to zero as $t \rightarrow \infty$, hence $\left\{u_{1}, u_{2}\right\}(\rho)=0$, and we have proved that $\mathscr{T}_{+}$is involutive. Studying the evolution of $T_{\rho}\left(\mathscr{T}_{t}\right)$ when $\rho \in \Sigma$ we easily see that $T_{\rho}\left(\mathscr{T}_{+}\right)=T_{\rho}(\Sigma) \oplus \Lambda_{+}$in the real domain.

\section{References}

[1] R. Abraham and J. MARSDEN : Foundations of Mechanics, Benjamin/Cummings publ. company 1978.

[2] L. Boutet de Monvel, A. Grigis and B. Helffer: Paramétrixes d'opérateurs pseudodifférentiels à caractéristiques multiples, Astérisque 34-35 (1976), 93121.

[3] A. GRIGIS, P. SChAPIRA and J. SJÖSTRAND: Propagation de singularités analytiques pour des opérateurs à caractéristiques multiples, C. R. A. S. 293 (2 nov. 1981), I-397-400.

[4] B. HELFFER: Invariants associés à une classe d'opérateurs pseudodifférentiels, Ann. Inst. Fourier, 26 (1976) no 2, 55-70.

[5] L. HÖRMANDER: The Weyl calculus of pseudodifferential operators, Comm. P. A. M., 32 (1979), 359-443.

[6] L. HÖRMANDER: Pseudodifferential operators and non-elliptic boundary problems, Ann. of Math. 83 (1966), 138-183.

[7] L. HÖRMANDER: A class of hypoelliptic pseudodifferential operators with double charactetistics, Math. Ann. 217 (1975), 165-188.

[8] M. KASHIWARA and T. KAWAI: Microhyperbolic pseudodifferential operators I, J. Math. Soc. Japan 27 (1975), 359-404.

[9] B. LASCAR and J. SJÖSTRAND: Equation de Schrödinger et propagation des singularités pour des opérateurs pseudodifférentiels à caractéristiques réelles de multiplicité variable II, A paraître.

[10] G. LEBEAU: A paraître.

[11] G. METIVIER: Analytic hypoellipticity for operators with multiple characteristics, Comm. in P. D. E., VI (1) (1981), 1-90.

[12] G. METIVIER: Non hypoellipticité analytique pour des opérateurs à caractéristiques doubles, Sém. Goulaouic-Meyer-Schwartz, 1981-82, no 12.

[13] T. ÔAKU: A canonical form of a system of microdifferential equations with non-involutory characteristics and branching of singularities, Inv. Math. 65 (1982), 491-525.

[14] J. SJÖSTRAND: Singularités analytiques microlocales, Astérisque, à paraître.

[15] J. SJÖSTRAND: Analytic singularities and microhyperbolic boundary value problems, Math. Ann. 254 (1980), 211-256.

[16] J. SJÖSTRAND: Analytic regularity for operators with multiple characteristics, Rendiconti del Sem. Mat. Univ. Polit. Torino, à paraître.

[17] D. TARTAKOFF: The local real analyticity of solutions to $\square b$ and the $\bar{\partial}$-Neumann problem, Acta Math. 145 (1980), 177-204. (See also Proc. Nat. Acad. Sci. U. S. A. 75 (1978), 3027-3028). 
[18] D. TARTAKOFF : Elementary proofs of analytic hypoellipticity for $\square_{b}$ and the $\bar{\partial}$-Neumann problem, Astérisque 89-90 (1981), 85-116.

[19] F. TRÈvES: Analytic hypoellipticity of a class of pseudodifferential operators with double characteristics, Comm. in P. D. E., 3 (1978), 475-642.

Département de Mathématiques

Université de Paris-Sud

Orsay, France 